\title{
Intrusion of UHP metamorphic rocks into the upper crust of Kyrgyzian Tien-Shan: $P$ - $T$ path and metamorphic age of the Makbal Complex
}

\author{
Michio TAgIRI ${ }^{*}$, Shingo TAKiguchI ${ }^{* *}$, Chika IshidA ${ }^{* * *}$, Takaaki NoguCHI", \\ Makoto KIMURA ${ }^{*}$, Apas BAKIROV ${ }^{\dagger}$, Kadyrbek SAKIEV ${ }^{\dagger}$, Masaki TAKAHASHI", \\ Akira TAKASU ${ }^{\S}$, Azamat BAKIROV ${ }^{\S}$, Asel TogONBAEVA ${ }^{\S}$ and Akio SUZUKI ${ }^{\#}$ \\ ${ }^{*}$ College of Sciences, Ibaraki University, Mito 310-8512, Japan \\ ${ }^{* *}$ Sakainishi High School, Sakai 306-0405, Japan \\ ${ }^{* * *}$ Toyosato Junior High School, Tsukuba 300-2642, Japan \\ Institute of Geology, Academy of Science, Republic Kyrgyz, \\ Erkendik pr. 30, 720481, Bishkek, Republic Kyrgyz \\ ${ }^{*}$ College of Humanities and Sciences, Nihon University, Setagayaku 156-8550, Japan \\ ${ }^{\S}$ Department of Geoscience, Interdisciplinary Faculty of Science and Engineering, \\ Shimane University, Matsue 690-8504, Japan \\ ${ }^{\#}$ Department of Mineralogy, Petrology and Economic Geology, Faculty of Science, \\ Tohoku University, Sendai 980-8578, Japan
}

\begin{abstract}
Coesite occurs in garnets from quartz schists and pelitic schists in the Makbal Complex of Kyrgyzian TienShan. The thick quartz schists interbedded with pelitic schists are the transitional facies after coesite schists. Quartz-pseudomorph after coesite also appears widely in pelitic schists and quartz schists, but is rare in eclogitic rocks. The growth zoning of garnets and $\mathrm{Na}-\mathrm{Ca}$ amphiboles in eclogites and the garnet-omphacite geothermometry indicate that the metamorphic $P-T$ paths of eclogites are different from each. Ultra-high (UHP) pressure metamorphic rocks of the Makbal Complex are mainly composed of pelitic and quartz schists. All eclogite lenses are included in the host UHPM rocks, although most of them do not contain UHP metamorphic (UHPM) evidences. The $\mathrm{K}-\mathrm{Ar}$ ages of phengite in a host pelitic schist and of paragonite in an eclogite are approximately $500 \mathrm{Ma}$, while those of biotite and phengite in a biotite-bearing mica schist and of winchite in a winchite schist are approximately 769-717 Ma and $881 \mathrm{Ma}$, respectively. The evidence indicates that this complex is a tectonic mélange. These geological relationships suggest that the ascending substances must be pelitic and quartz schists. The UHPM rocks in the ascending material capture many exotic blocks as xenoliths during intrusion into the upper crust.
\end{abstract}

Keywords: Coesite schist, Tectonic intrusion, Ascending substance, $P-T$ path, Isotopic age, Tien-Shan, Kyrgyzstan

\section{INTRODUCTION}

The geological relationship between ultra-high pressure (UHP) pelitic schists and eclogites has been reported in many studies; however, there have been limited discussions on the ascending carrier (e.g., England and Holland, 1979; Platt and Wallis, 1991; Zhang et al., 1997; Kaneko et al., 2000). It seems that UHP metamorphic (UHPM) rocks rise rapidly from the upper mantle to the crust.

doi:10.2465/jmps.071025

M. Tagiri, tagiri@mx.ibaraki.ac.jp Corresponding author
Hacker and Peacock (1995) discussed the exhumation rate of UHPM rocks and reported the rate of Dora-Maira as being $3 \mathrm{~mm} /$ year. They also emphasized that fluid should proceed for the transformation from coesite to quartz during exhumation. In contrast to the study of Hacker and Peacock (1995), three papers-by Gebauer et al. (1997), Duchêne et al. (1997), and Rubatto and Hermann (2001) - have reported that the exhumation rate of Dora-Maira is $20-30 \mathrm{~mm} /$ year. According to Zhang et al. (1997) and Hermann et al. (2001), Kokchetav UHPM rocks rose at a rate of approximately $10-30 \mathrm{~mm} /$ year. Do- 
bretsov and Shatsky (2004) proposed extremely high uplift velocities of 60-200 mm/year for the Kokchetav UHPM rocks; however, the exhumation mechanism has not yet been understood completely. Rubatto and Hermann (2001) and Hermann et al. (2001) have suggested that fast exhumation is driven by a combination of buoyancy and normal faulting. For example, England and Holland (1979) proposed that exhumation of the Tauern eclogites is a result of the buoyancy of carbonaceous rock. Dobretsov and Shatsky (2004) proposed a corner flow model for superfast exhumation. Detailed discussion regarding the ascending mechanism of UHPM rocks is still required, especially that based on field observations.

Tagiri and Bakirov (1990) and Tagiri et al. (1995) have reported the existence of coesite pseudomorph-bear-
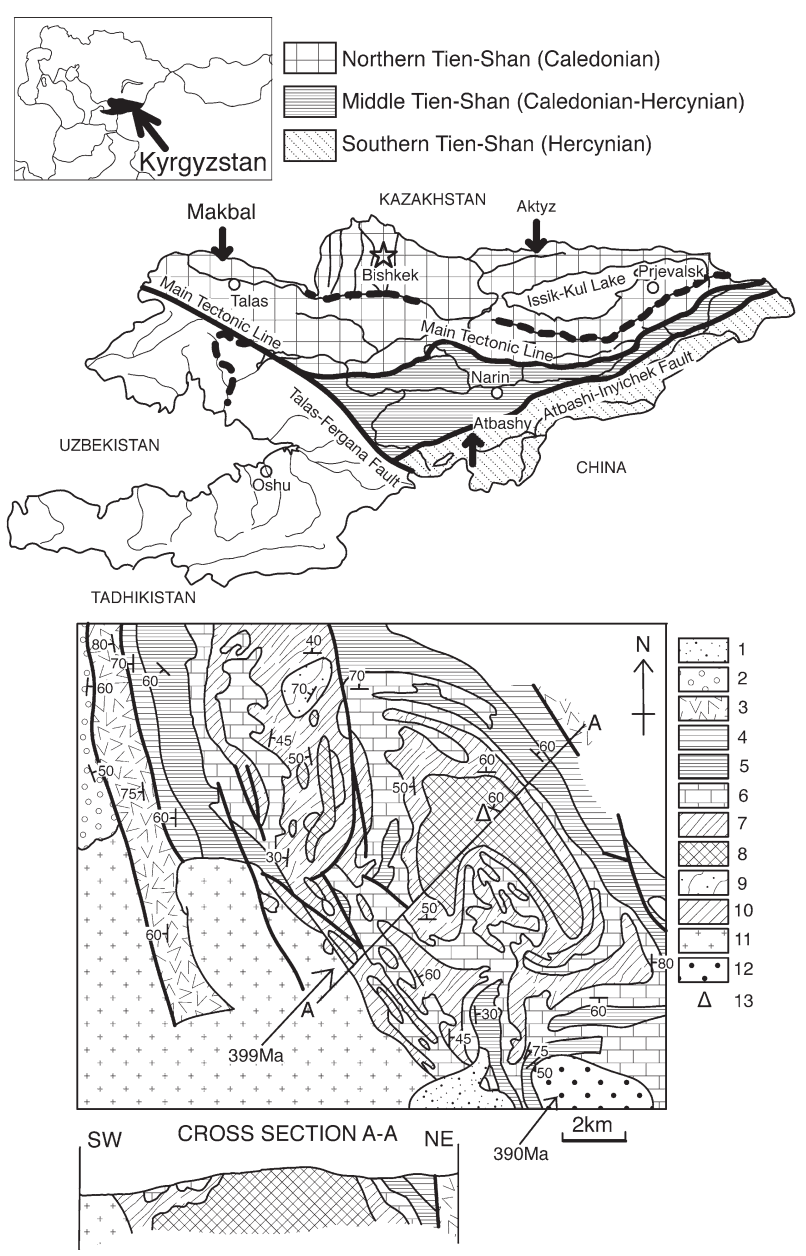

Figure 1. Index map and geological map of the Makbal district in Kyrgyzstan. 1, Quaternary formation; 2, Ordovician formation; 3, Lower Paleozoic formation; 4-6, Scharkurak Group metamorphics, 4-5, Mica schists; 6, Marble; 7-9, Akdzhon Group metamorphics: 7, Neldy metamorphics; 8 , UHP rock-bearing Makbal Complex; 9, Mylonitized Lower Proterozoic granite, 10, Basic schists; 11, Intrusive granites; 12, Karajulga granites; 13, The area of Figures 2 and 3. ing UHPM rocks in two localities in the Kyrgyzian TienShan. In this paper, we will report on relic coesite-bearing pelitic and quartz schists and associated rocks of the Makbal Complex. Coesite-bearing pelitic schists are intercalated with coesite-bearing quartz schists. The contact relationship between the host UHP schists and the eclogite lenses can be clearly observed in the Makbal district. Eclogite lenses are included in these pelitic and quartz schists, and the chemical compositions of garnets and $\mathrm{Na}^{-}$ $\mathrm{Ca}$ amphiboles in eclogites have variations in each sample. Geological evidence suggests that the Makbal Complex is a tectonic mélange (Bakirov et al., 1998). On the basis of these geological observations, the isotopic age data, and the mineralogical data, we propose an ascent mechanism for UHPM rocks and high-pressure (HP) eclogites.

\section{GEOLOGICAL OUTLINE OF THE MAKBAL COMPLEX}

The Tien-Shan (mountains) is a part of the Paleozoic Ural-Mongolian fold belt (Bakirov et al., 1984; Sobolev et al., 1986). The Kyrgyzian Tien-Shan consists of three geotectonic zones (Fig. 1): the Caledonian fold zone (Northern Tien-Shan), the Caledonian-Hercynian fold zone (Middle Tien-Shan), and the Hercynian fold zone (Southern Tien-Shan). Distinct tectonic lines separate these three zones from each other.

The Makbal eclogite-bearing complex occurs in the Caledonian fold belt extending through Central Kazakhstan (Bakirov et al., 1998) and the southern Urals (Chemenda et al., 1997). The Makbal district is located in the western part of the Kyrgyz Range, and UHPM rocks can be found in both Kyrgyzstan and Kazakhstan.

The Makbal Complex is mainly composed of pelitic schists and quartz schists alternated with calcareous schists. Eclogites and amphibolites retrograded from eclogite occur in the host pelitic schists and quartz schists as imbricated lenses of different sizes ranging from 0.1 to $50 \mathrm{~m}$ in diameter (Figs. 2, 3, 4a, 4c, and 4e). As described later, two types of eclogites occur as lenses: UHP and HP. Distinct slip planes commonly exist between eclogite lenses and the host rocks. A drag fold also develops around amphibolitized eclogite lenses (Fig. 4a). Moreover, garnet porphyroclasts in eclogite have an S-shape deformation structure (Fig. 5a). On the other hand, parallel foliation and folds are common in pelitic schists and quartz schists. These geological evidences indicate that eclogite lenses were highly imbricated after their juxtaposition with the host rocks and that the deformation continued after the juxtaposition.

The Makbal Complex has primarily undergone HP 


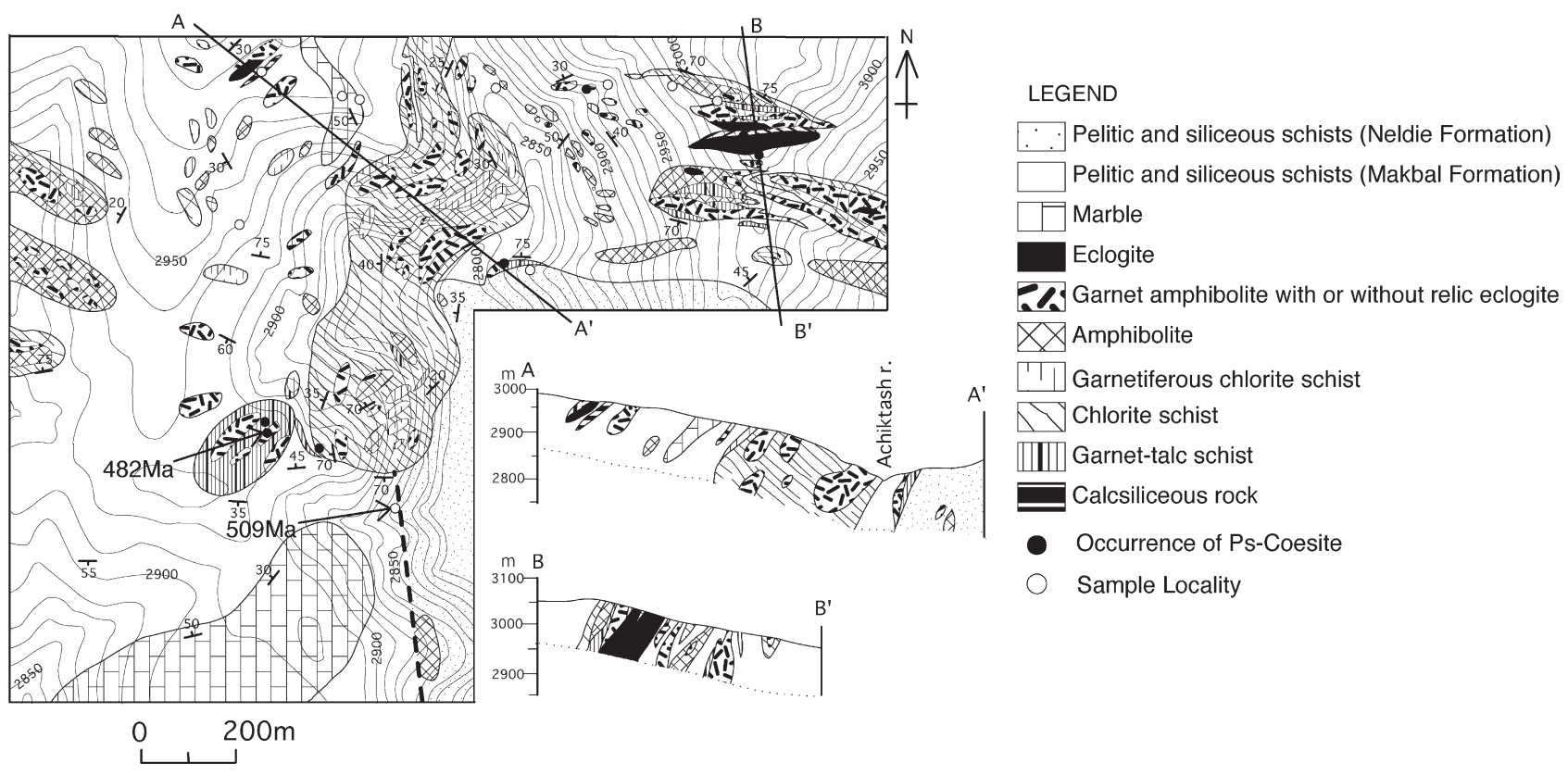

Figure 2. Geological map of the Achiktash river area (Sobolev et al., 1986).

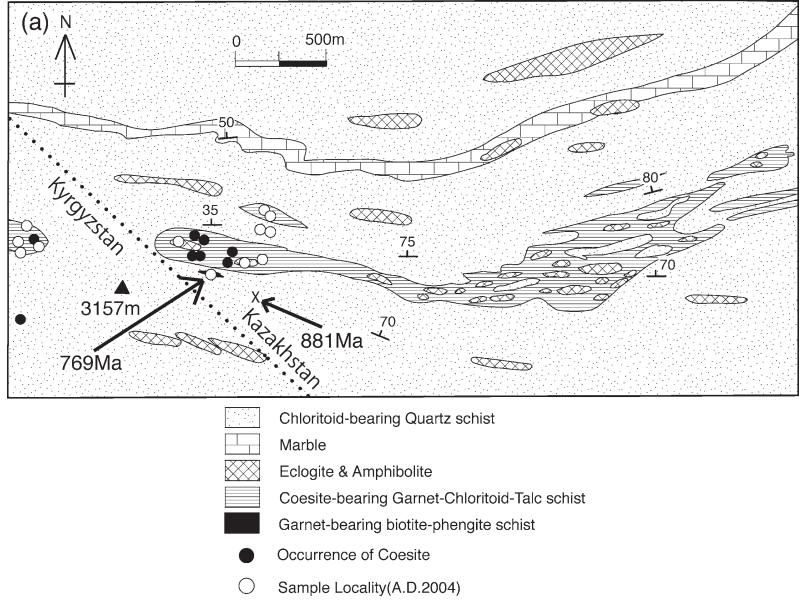

(b)

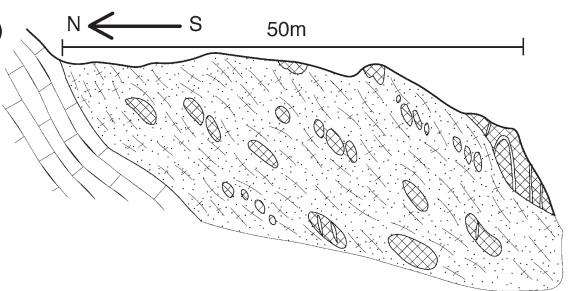

(c)

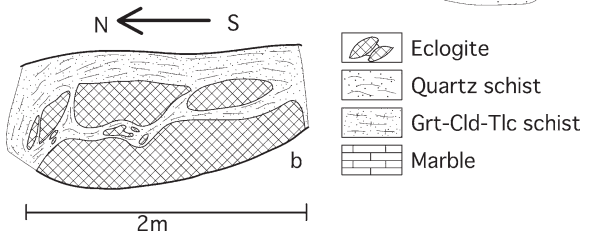

Figure 3. (a) Geological map of the summit area (Bakirov et al., 1998). (b) and (c) Sketches of imbricate eclogites in pelitic schists. The sketches show eclogite lenses in schists of the Makbal Complex. Eclogite bodies imbricate as lenses. and UHP metamorphism and has retrogressively transformed to epidote-amphibolite facies rocks (Tagiri et al., 1995). However, the amphibolitization of eclogites does not depend on the size of the eclogite lens. All samples in this study were taken from the Achiktash river area and the summit area (Figs. 2 and 3).

The protolith age of the Makbal Complex is unknown as there are some geological constraints for determining the age of the Makbal Complex. The $\mathrm{K}-\mathrm{Ar}$ age of paragonite associated with garnet and omphacite in eclogite is $482 \pm 12 \mathrm{Ma}$ (Table 1 and Fig. 2) (Tagiri et al., 1995). The CHIME age of monazite in garnet-chloritoidtalc schists is $481 \pm 26$ Ma from the matrix of the schists and $480 \pm 56 \mathrm{Ma}$ from the inclusion in garnet (Togonbaeva et al., 2009). Togonbaeva et al. (2009) suggested these ages for the UHP metamorphism in the Makbal Complex. Two granitic bodies intruding into the Makbal Complex have $\mathrm{K}-\mathrm{Ar}$ ages of orthoclase, indicating Devonian origin (Table 1 and Fig. 1).

\section{COESITE-BEARING SCHISTS}

\section{Coesite-bearing pelitic schists and quartz schists}

Thick coesite-bearing quartz schists in the Makbal complex contain a small amount of coesite-bearing pelitic garnet-chloritoid-talc schists (Figs. 3a, 4d, 5c, and 5d). The quartz schists and pelitic schists are coarse-grained, and the grain size of quartz and garnet reaches more than $10 \mathrm{~mm}$ in diameter. Retrograde reaction is indistinct in these schists, except for the transformation from coesite 

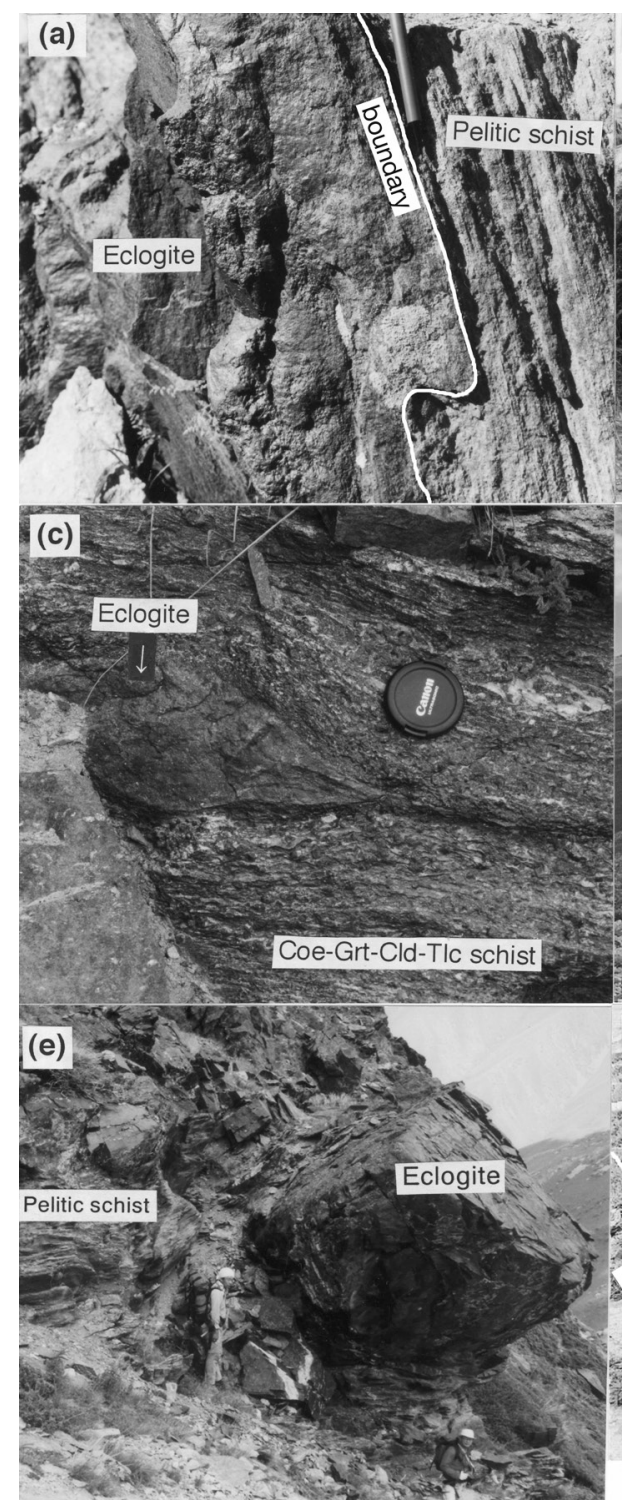

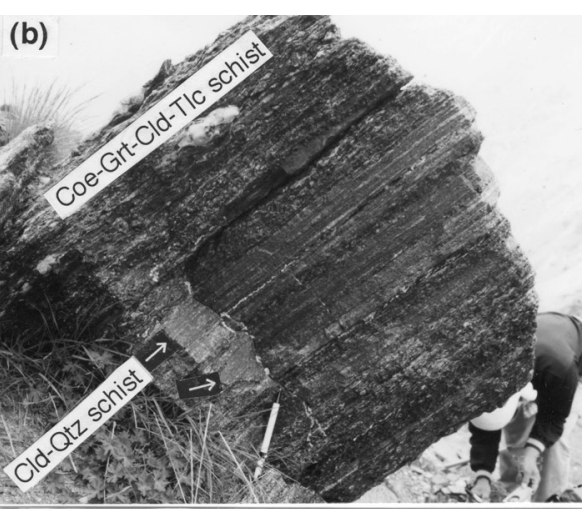

(d)
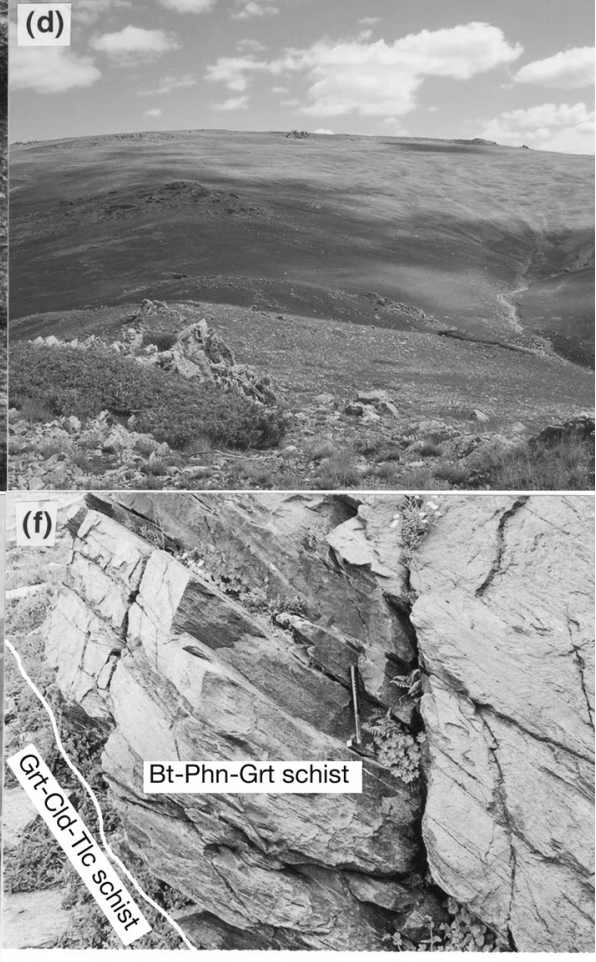

Figure 4. Outcrop photographs in the summit area. (a) Deformation of amphibolitized eclogite lens in a pelitic schist. A drag fold is indicated with a white line. (b) Alternation of coesite-bearing pelitic schists and quartz schists. (c) A small eclogite lens in coesite-bearing pelitic schist. (d) Wide development of coesite-bearing quartz schists in the summit area. Quartz schists cover the entire scope of the photograph. (e) A large lenticular block of eclogite included in the alternation of pelitic and quartz schists. (f) An exotic block of biotite-phengite-garnet schist (sample 94071602). to quartz. Coesite has been determined using laser Raman spectroscopy (NRS2000) at Tohoku University (Fig. 6). A $514.73 \mathrm{~nm}$ laser operating at $12 \mathrm{~mW}$ was used with an exposure time of $300 \mathrm{~s}$. Thick quartz schists containing garnet porphyroblast in which coesite and/or coesite-pseudomorph is included were observed sporadically. Coesite and coesite-pseudomorph appear widely in the pelitic schists (Figs. 2 and 3). Quartz in the matrix of the pelitic schists must have been coesite during the UHPM stage. Similarly, the thick quartz schists associated with UHP pelitic schists must have undergone UHPM conditions, and the silica mineral of quartz schist was once coesite. That is, quartz schist is a transformation facies after coesite schist in this complex.

Tables 2 and 3 give the mineral assemblage and chemical compositions of the constituent minerals in the pelitic and quartz schists. The mineral assemblage of the
UHPM stage of sample 1609-KYR18 is coesite + garnet + chloritoid + talc + phengite + outer-core and rim of tourmaline. Coesite typically remains as inclusions in garnet (Fig. 5b). The assemblage of the matrix is garnet + chloritoid + talc + phengite + glaucophane + outer-rim of tourmaline + quartz (Fig. 5c). The garnet in this sample does not contain glaucophane, but glaucophane is closely associated with other minerals in the matrix. The direct contact of glaucophane and talc in Figure 5c suggests the possibility that the glaucophane + talc assemblage was retrogressively formed from jadeite in the UHPM stage (Massonne, 1995), although jadeite does not occur in this sample. The prograde garnet has a weak zonal structure (93063005). The chemical composition of chloritoid associated with coesite in garnet is not very different from that of the chloritoid in the matrix, except for a slight variation in the $\mathrm{Fe} / \mathrm{Mg}$ ratio (Table 3). 

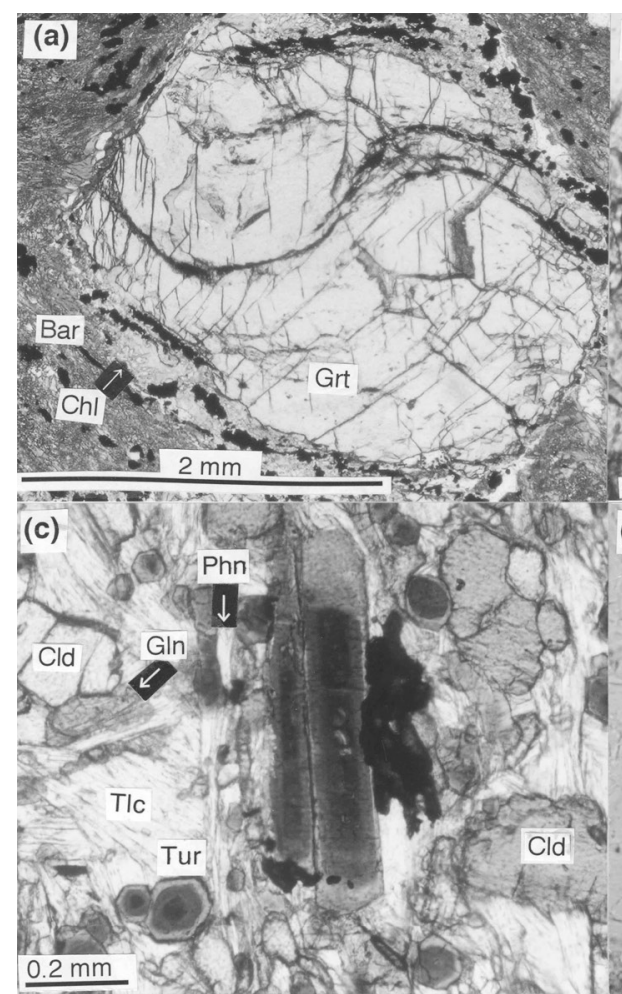

(b)
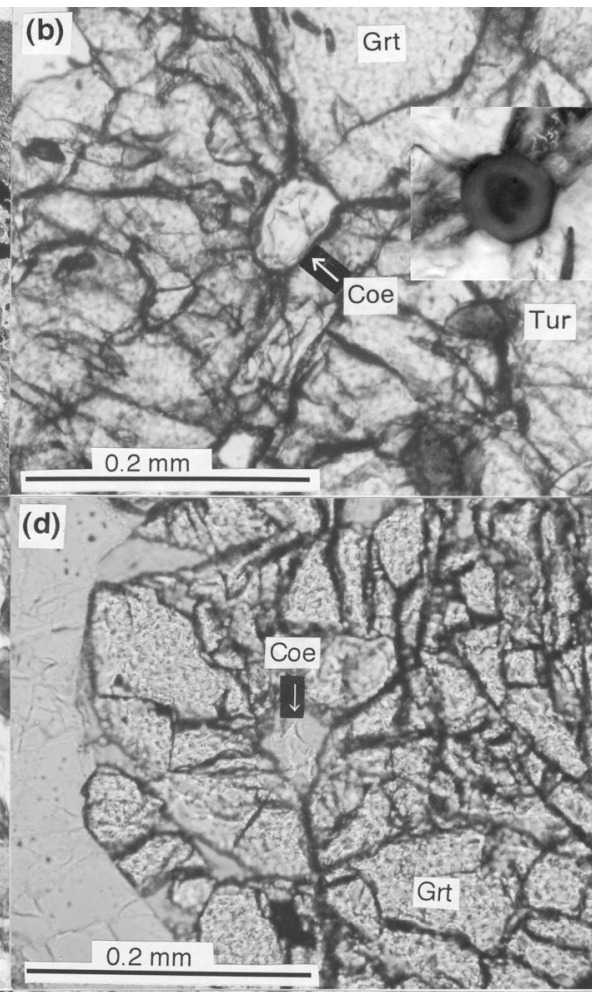

(d)
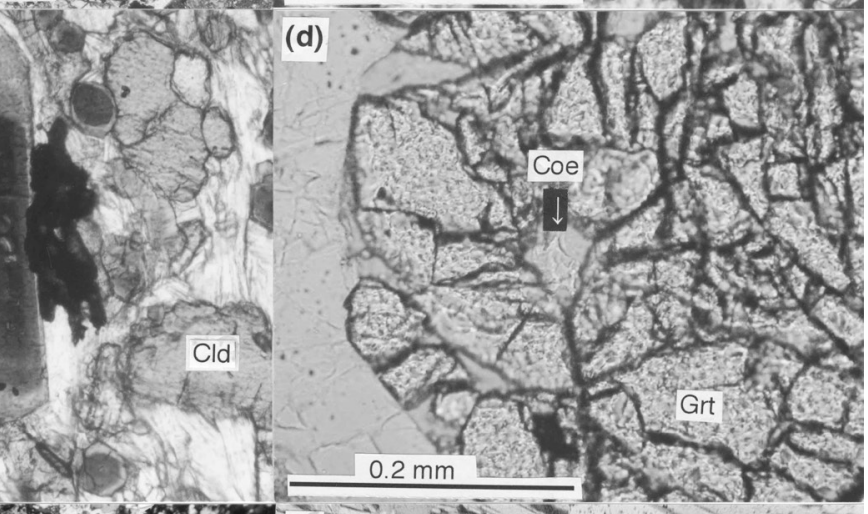

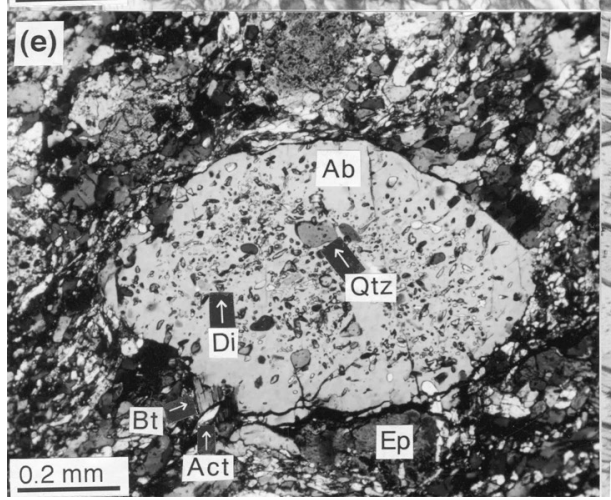

(f)
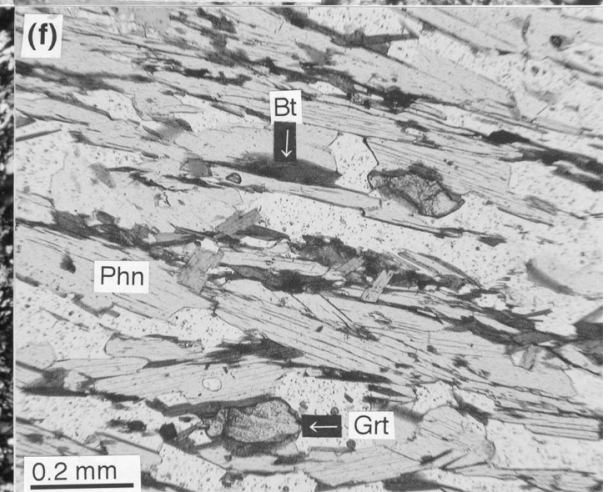

Figure 5. Microphotographs of coesitebearing pelitic schist and calc-siliceous schist. (a) Amphibolitized eclogite including type III garnet porphyroclast, indicated by the S-shaped deformation (93070106A). Bar, barroisite. (b) Coesite inclusion in garnet from pelitic schist (1609-KYR18). The pleochroic color of zoned tourmaline in garnet is similar to that of the tourmaline in the matrix. The scale of the window is twice that of the main photo. (c) Zonal structure of tourmaline in a matrix of coesite-bearing pelitic schist (1609-KYR18). Note the close association of glaucophane + talc and chloritoid + tourmaline. (d) Coesite inclusion in garnet from quartz schist (4080807). (e) Albite porphyroblast in an exotic block of calc-siliceous schist (93070102), viewed with crossed nicols. Sodic diopside and quartz are included in the porphyroblast. (f) An exotic block of biotite-phengite-garnet schist (717-769 Ma).

Table 1. K-Ar ages of the Makbal Complex and intrusive granites

\begin{tabular}{cccccccc}
\hline & & Material & \multicolumn{5}{c}{ Ar-40(rad.) } \\
Sample No. & Rock name & analysed & K-Ar age (Ma) & $\left(\mathrm{scc} / \mathrm{g} \times 10^{-5}\right)$ & $\%$ Ar-40(rad.) & $\% \mathrm{~K}$ & Reference \\
\hline $82-2641$ & Eclogite & paragonite & $482 \pm 17$ & 11.1 & 62.3 & 0.623 & Tagiri et al. (1995) \\
KG17 & Grt+Cld+Phn schist & phengite & $509 \pm 13$ & 13.9 & 97.2 & 6.09 & This study \\
94071602 & Bt+Phn+Grt schist & biotite & $769 \pm 19$ & 22.3 & 98.7 & 5.93 & This study \\
94071602 & Bt+Phn+Grt schist & phengite & $717 \pm 18$ & 25 & 97.9 & 7.28 & This study \\
93063015 & winchite schist & winchite & $881 \pm 22$ & $0.793,0.797$ & $91.6,92.4$ & $0.18,0.18$ & This study \\
Intrusive granite & granite & orthoclase & $399 \pm 10$ & 9.09 & 95.6 & 5.26 & This study \\
Karajurga granite & granite & orthoclase & $389 \pm 8$ & 17.2 & 99.1 & 10.22 & This study \\
\hline
\end{tabular}

Five measurements have been done by Geospace Science Co.

Karajurga granite dating was made by Mitsubishi Materials Corporation

Although the garnet from the coesite-bearing pelitic schists has no distinct compositional growth zoning, tourmaline shows distinct growth zoning, as revealed by optical (Figs. 5b and 5c) and compositional examination.
Tourmaline in garnet has three zones: the euhedral rim, euhedral outer core, and the anhedral inner core. The shape and chemical composition of the anhedral inner core suggest that it may be a sedimentary relict. The euhe- 

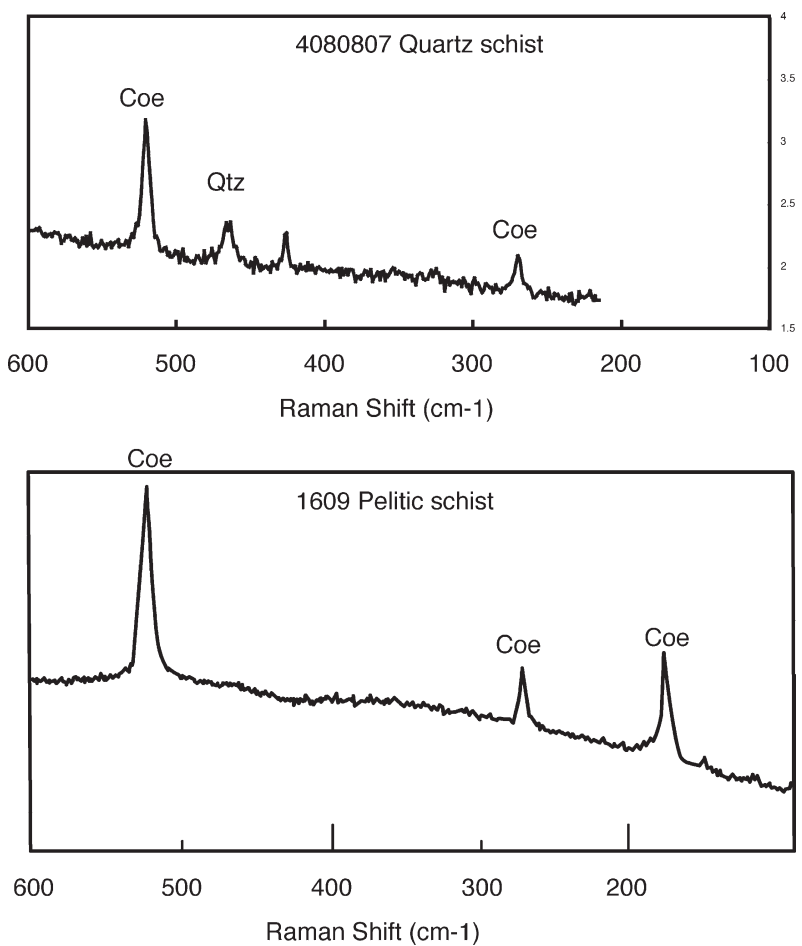

Figure 6. Laser Raman spectroscopy of coesites in a pelitic schist (1609-KYR18) and in a quartz schist (4080807). The Raman spectra fit well with the spectra of coesite (Liu et al., 1997).

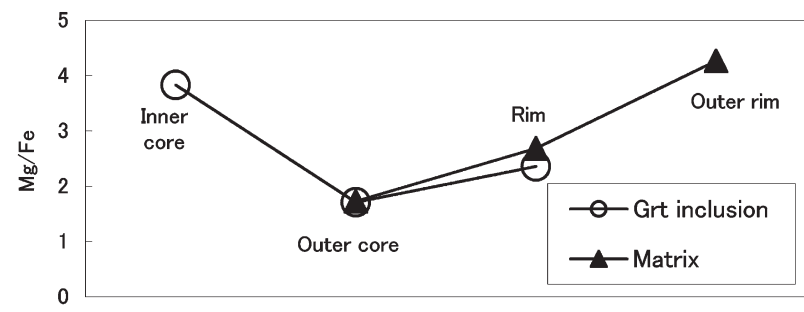

Figure 7. Compositional growth zoning of tourmaline.

dral rim grows over the euhedral outer core. The tourmaline in the matrix has four zones (Fig. 5b). From the inner core to the rim of the matrix tourmaline, the optical and chemical features are very similar to those of tourmaline in the garnet. The fourth zone, i.e., the outer rim, grows over the rim of matrix tourmaline during the later stage. The outer core and the rim of the tourmaline in garnet and the tourmaline in the matrix are crystallized at the same stage under UHPM conditions, which implies that garnet porphyroblast including coesite is not an accidental xenocryst in sedimentary rocks. The outer rim of matrix tourmaline is associated with garnet, glaucophane, talc, and chloritoid in the later stage.

The UHPM of the Makbal Complex is a low temperature type, as the chloritoid is associated with coesite

Table 2. Mineral assemblage of the Makbal Complex

\begin{tabular}{|c|c|c|c|c|c|c|c|c|c|c|c|c|c|}
\hline Pelitic and Qtz schist & $\mathrm{Coe}$ & Grt & Cld & Tlc & W-M & $\mathrm{Bt}$ & Amp & $\mathrm{Rt}$ & Tur & Qtz & Chl & Flo & $\mathrm{Ab}$ \\
\hline $\mathrm{Mb}^{*}$ & Ps & ++ & ++ & ++ & $\mathrm{Phn}$ & $(+)$ & & + & & $(+)$ & $(+)$ & $(+)$ & $(+)$ \\
\hline 93063005 & + & ++ & ++ & ++ & $\mathrm{Phn}$ & $(+)$ & & + & & $(+)$ & $(+)$ & $(+)$ & $(+)$ \\
\hline $93063007 a$ & & & ++ & & $\mathrm{Phn}$ & & & + & & $(++)$ & $(+)$ & & $(+)$ \\
\hline 93070101 & & & ++ & & $\mathrm{Phn}$ & $(+)$ & & & + & $(++)$ & $(+)$ & & $(+)$ \\
\hline 94071605 & & ++ & ++ & ++ & & & & + & & $(++)$ & $(+)$ & & $(+)$ \\
\hline $1609-\mathrm{KYR} 18^{* * * *}$ & + & + & + & + & $\mathrm{Phn}$ & $(+)$ & $(\mathrm{Na})$ & + & + & $(++)$ & $(+)$ & & $(+)$ \\
\hline 4080807 & + & + & + & + & $\mathrm{Phn}$ & & & + & & $(+++)$ & & & \\
\hline 94071602 & & + & & & $\mathrm{Phn}$ & ++ & & & & ++ & & & + \\
\hline Calc-siliceous schist & Ep & Cpx & Amp & $\mathrm{Pl}$ & W-M & $\mathrm{Bt}$ & Kfs & $\mathrm{Rt}$ & Tur & Qtz & Chl & Spn & $\mathrm{Cbn}$ \\
\hline $93070102^{* * * *}$ & + & $\mathrm{Di}$ & Act & Ab-spot & Phn & + & + & + & + & ++ & + & + & + \\
\hline 93070202 & + & & Act & & & & & + & & ++ & + & + & + \\
\hline 93070204 & & & Act & & & & & + & & ++ & + & + & + \\
\hline Eclogite & Coe & Grt & $\mathrm{Cpx}$ & Na-Ca Amp & Ep & Zo & W-M & $\mathrm{Bt}$ & Rt & Qtz & Spn & Pl & Chl \\
\hline $34-53 \mathrm{~A}$ & & $8 \mathrm{mII}$ & Omp & $\operatorname{Bar}(++)$ & + & & $\mathrm{Phn}, \mathrm{Pg}$ & & + & + & & $(+)$ & $(+)$ \\
\hline $82-2641^{* *}$ & & $5 \mathrm{III}$ & Omp & $(++)$ & + & & $\mathrm{Pg}$ & & + & + & $(+)$ & $(+)$ & $(+)$ \\
\hline $93062901 \mathrm{~B}$ & & $13 \mathrm{mI}$ & Omp & $(++)$ & + & & $\mathrm{Pg}$ & & + & + & $(+)$ & $(+)$ & \\
\hline $93070106 \mathrm{~A}$ & Ps & $8 \mathrm{III}$ & (sym) & $\mathrm{Gln}(++)$ & ++ & & $(\mathrm{Pg})$ & & & $(+)$ & & $(+)$ & $(+)$ \\
\hline 93070110 & Ps & 9I & Omp & $(++)$ & + & & $(\mathrm{Pg})$ & & + & $(+)$ & $(+)$ & $(+)$ & \\
\hline $94071601^{* * *}$ & & $2 \mathrm{mIII}$ & Omp & $(++)$ & + & & $\mathrm{Phn}, \mathrm{Pg}$ & & + & + & & $(+)$ & \\
\hline $94071606^{* * *}$ & & 1II & Omp & $(++)$ & + & & $\mathrm{Pg}$ & & + & + & + & $(+)$ & $(+)$ \\
\hline 94071610 & & $3 \mathrm{mI}$ & Omp & $\operatorname{Trm}, \operatorname{Gln}(++)$ & ++ & + & $\mathrm{Pg}$ & + & + & + & & $(+)$ & \\
\hline Winchite schist (930 & & & & +++ & & & & & & & & + & \\
\hline
\end{tabular}

Abbreviations follow Edit. Comm., G.S.J. (2000). W-M, white micas; Flo, florencite; Ps, pseudomorph; Cpx, clinopyroxene; Na-Ca Amp, sodic and calcic amphiboles; parentheses, retrogressive secondary mineral; sym, Pl-Act symplectite; *, Tagiri and Bakirov (1990); ${ }^{* *}$, Tagiri et al. (1995); ${ }^{* * *}$, Puelles and Takasu (1996); ${ }^{* * * *}$, Bakirov et al. (1998).

The numerals, $\mathrm{m}$ and I-III of Grt column indicate the grain number of analyzed garnet, mapping analysis and zoning type of I to III, respectively. 
Table 3. Chemical compositions of representative minerals

\begin{tabular}{|c|c|c|c|c|c|c|c|c|c|c|c|c|c|c|c|c|}
\hline Sample & \multicolumn{5}{|c|}{93070102} & \multicolumn{4}{|c|}{93070202} & \multicolumn{2}{|c|}{93063005} & \multicolumn{5}{|c|}{ 1609-KYR18 } \\
\hline Rock & Calc-si & liceous & schist & & & Calc-sil & liceou & schist & & Pelitic s & chist & Pelitic & schist & & & \\
\hline $\begin{array}{l}\text { Mineral } \\
\text { comment }\end{array}$ & $\mathrm{Di}$ & Amp & $\mathrm{Phn}$ & $\mathrm{Phl}$ & Kfs & Tlc & Amp & Spn & Dol & Grt & Grt & Grt & $\begin{array}{c}\text { Cld } \\
\text { grt -incl }\end{array}$ & $\begin{array}{c}\text { Cld } \\
\text { matrix }\end{array}$ & $\begin{array}{c}\text { Gln } \\
\text { matrix }\end{array}$ & Phn \\
\hline position & & & & & & & & & & core & rim & & & & & \\
\hline$\overline{\mathrm{SiO}_{2}}$ & 53.56 & 56.46 & 54.37 & 40.92 & 62.75 & 60.28 & 57.22 & 29.83 & 0.19 & 37.19 & 37.74 & 36.05 & 22.90 & 24.64 & 57.50 & 49.9 \\
\hline $\mathrm{TiO}_{2}$ & 0.00 & 0.00 & 0.11 & 0.44 & 0.00 & 0.00 & 0.00 & 35.80 & 0.02 & 0.00 & 0.00 & 0.11 & n.d. & n.d. & n.d. & 0.17 \\
\hline $\mathrm{Al}_{2} \mathrm{O}_{3}$ & 1.81 & 1.14 & 25.96 & 13.70 & 18.54 & 0.26 & 1.68 & 2.56 & 0.00 & 21.10 & 21.37 & 20.54 & 37.80 & 40.35 & 11.27 & 26.59 \\
\hline $\mathrm{FeO}$ & 8.21 & 8.25 & 2.12 & 11.14 & 0.06 & 4.06 & 3.38 & 0.00 & 3.25 & 33.84 & 31.44 & 32.81 & 21.70 & 20.37 & 5.69 & 1.85 \\
\hline $\mathrm{MnO}$ & 0.43 & 0.44 & 0.13 & 0.10 & n.d. & 0.04 & 0.06 & 0.03 & 0.22 & 1.35 & 0.25 & 0.33 & 0.04 & 0.10 & 0.07 & 0.00 \\
\hline $\mathrm{MgO}$ & 11.86 & 17.99 & 4.00 & 18.86 & 0.01 & 28.15 & 21.40 & 0.01 & 18.98 & 3.56 & 7.18 & 4.83 & 4.34 & 5.60 & 11.63 & 3.52 \\
\hline $\mathrm{CaO}$ & 22.70 & 13.05 & 0.05 & 0.00 & 0.00 & 0.16 & 12.32 & 29.32 & 29.52 & & 0.75 & 3.30 & n.d. & n.d. & 26 & 0.03 \\
\hline $\mathrm{Na}_{2} \mathrm{O}$ & 1.46 & 0.36 & 0.14 & 0.07 & 0.70 & 0.10 & 0.88 & 0.00 & 0.04 & 0.05 & 0.10 & n.d. & 0.25 & 0.24 & 6.57 & 0.45 \\
\hline $\mathrm{K}_{2} \mathrm{O}$ & 0.14 & 0.10 & 10.25 & 9.41 & 15.05 & 0.00 & 0.11 & 0.00 & 0.02 & 0.00 & 0.00 & n.d. & n.d. & n.d. & n.d. & 11.02 \\
\hline $\mathrm{BaO}$ & n.d. & n.d. & n.d. & n.d. & 2.56 & n.d. & n.d. & n.d. & n.d. & n.d. & n.d. & n.d. & n.d. & n.d. & n.d. & n.d. \\
\hline Total & 100.17 & 97.81 & 97.13 & 94.64 & 99.67 & 93.06 & 97.04 & 97.55 & 52.24 & 99.54 & 98.84 & 97.98 & 87.03 & 91.30 & 92.99 & 93.60 \\
\hline Cation & $\mathrm{O}=6$ & $\mathrm{O}=23$ & $\mathrm{O}=11$ & $\mathrm{O}=11$ & $\mathrm{O}=8$ & $\mathrm{O}=11$ & $\mathrm{O}=23$ & $\mathrm{O}=4$ & $\mathrm{O}=6$ & $\mathrm{O}=12$ & $\mathrm{O}=12$ & $\mathrm{O}=12$ & $\mathrm{O}=12$ & $\mathrm{O}=12$ & $\mathrm{O}=23$ & $\mathrm{O}=11$ \\
\hline $\mathrm{Si}$ & 2.00 & 7.99 & 3.53 & 3.00 & 2.96 & 3.98 & 7.92 & 0.80 & 0.01 & 2.99 & 2.99 & 2.94 & 2.00 & 2.02 & 8.08 & 3.40 \\
\hline $\mathrm{Ti}$ & 0.00 & 0.00 & 0.01 & 0.02 & 0.00 & 0.00 & 0.00 & 0.72 & 0.04 & 0.00 & 0.00 & 0.01 & - & - & - & 0.01 \\
\hline $\mathrm{Al}(\mathrm{IV})$ & 01 & 0.01 & 0.47 & 1.00 & 1.03 & 0.02 & 0.08 & 0.08 & 0.00 & 0.01 & 0.01 & 0.06 & - & - & 0.00 & 0.60 \\
\hline $\mathrm{Al}(\mathrm{VI})$ & 0.08 & 0.18 & 1.52 & 0.18 & - & - & 0.19 & 0.00 & - & 1.99 & 1.99 & 1.92 & 3.89 & 3.9 & 1.87 & 1.53 \\
\hline $\mathrm{Fe}^{\prime \prime \prime}$ & 0.04 & - & - & - & 0.00 & - & - & - & - & 0.01 & 0.01 & 0.08 & - & - & 0.13 & - \\
\hline $\mathrm{Fe}^{\prime \prime}$ & 0.22 & 0.74 & 0.12 & 0.68 & - & 0.22 & 0.38 & - & 0.08 & 2.27 & 2.08 & 2.16 & 1.59 & 1.40 & 0.54 & 0.10 \\
\hline $\mathrm{Mn}$ & 0.01 & 0.05 & 0.01 & 0.01 & - & 0.00 & 0.01 & 0.00 & 0.01 & 0.09 & 0.02 & 0.02 & 0.00 & 0.01 & 0.01 & 0.00 \\
\hline $\mathrm{Mg}$ & 0.66 & 3.79 & 2.06 & 2.06 & 0.00 & 2.77 & 4.41 & 0.00 & 0.87 & 0.43 & 0.85 & 0.59 & 0.57 & 0.69 & 2.44 & 0.36 \\
\hline $\mathrm{Ca}$ & 0.91 & 1.98 & 0.00 & 0.00 & 0.00 & 0.01 & 1.83 & 0.84 & 0.95 & 0.21 & 0.06 & 0.29 & - & - & 0.04 & 0.00 \\
\hline $\mathrm{Na}$ & 0.11 & 0.10 & 0.02 & 0.01 & 0.06 & 0.01 & 0.24 & 0.00 & 0.00 & 0.01 & 0.02 & - & 0.04 & 0.03 & 1.79 & 0.06 \\
\hline K & 0.01 & 0.02 & 0.85 & 0.88 & 0.91 & 0.00 & 0.02 & 0.00 & 0.00 & 0.00 & 0.00 & - & - & - & - & 0.96 \\
\hline B & - & - & - & . & 0.05 & - & - & - & - & - & - & - & - & - & - & - \\
\hline Total & 4.02 & 14.86 & 6.90 & 7.83 & 5.01 & 7.02 & 15.07 & 2.44 & 1.95 & 8.01 & 8.02 & 8.07 & 8.08 & 8.05 & 14.90 & 7.02 \\
\hline
\end{tabular}

$\mathrm{Fe}^{\prime \prime \prime}$ of Cpx is calculated as per the method given in Banno (1959). $\mathrm{Fe}^{\prime \prime \prime}$ of Amp is calculated when there are 5 cations at the $\mathrm{Y}$ site. Incl, inclusion; f-matrix, fine-grained matrix.

(Carswell, 1990). The upper stability limit of chloritoid in the iron-bearing system is about $560{ }^{\circ} \mathrm{C}$ in the coesite field and about $600{ }^{\circ} \mathrm{C}$ in the quartz field (Wei and Song, 2008) (see Fig. 14). The reaction garnet + carphorite $=$ chloritoid + talc, which has a positive sole in the KFMASH system, intersects with the coesite-quartz boundary at $\sim 550{ }^{\circ} \mathrm{C}$ (Wei and Powell, 2003). Massonne determined the Si content of phengite in the pelitic schist of the Makbal Complex to be 3.42 for $\mathrm{O}=11$ basis [Fig. 12a of Massonne and Schreyer (1989)]. Based on the phengite geobarometry of the talc + phengite + phlogopite + kyanite + silica mineral assemblage, Massonne and Schreyer (1989) estimated the metamorphic pressure to be $2.5 \mathrm{GPa}$ at $600{ }^{\circ} \mathrm{C}$. However, phengite in our samples (e.g., 1609KYR18) is not associated with phlogopite and kyanite. As a result, we estimated a metamorphic pressure of $2.8 \mathrm{GPa}$ at $\sim 560{ }^{\circ} \mathrm{C}$ for the Makbal Complex. The chloritoid + talc assemblage in pelitic schists, which are widely found in the Makbal Complex, should be stable during the early stage of exhumation at high pressure.

The glaucophane + chloritoid + talc + garnet assemblage occurs in the matrix of the sample 1609-KYR18, which is a Ca-poor pelitic schist. We could not observe any petrographic evidence for an UHPM assemblage in the matrix. Wei and Song (2008) studied the phase equilibrium of glaucophane + chloritoid. According to their results, the stability field of this assemblage is located outside of the lower temperature limit of chloritoid + talc (see Fig. 14) in the coesite field. However, the temperature difference between the two reactions is very small. Moreover, the reaction of garnet + carphorite + jadeite $=$ chloritoid + glaucophane studied by Wei and Song (2008) is inconsistent with that studied by Wei and Powell (2004). In the quartz field, the glaucophane + chloritoid assemblage is stable in a wide $P-T$ range. The chloritoid + talc assemblage is also widely stable in the range 
Table 3. (Continued 1)

\begin{tabular}{|c|c|c|c|c|c|c|c|c|c|c|}
\hline \multicolumn{6}{|l|}{ Sample } & \multicolumn{5}{|c|}{94071602} \\
\hline Rock & & & & & & Bt-Phr & n-Grt sc & chist & & \\
\hline $\begin{array}{l}\text { Mineral } \\
\text { comment } \\
\text { position }\end{array}$ & $\begin{array}{c}\text { Tur } \\
\text { grt-incl } \\
\text { outer core }\end{array}$ & $\begin{array}{c}\text { Tur } \\
\text { grt-incl } \\
\text { rim }\end{array}$ & $\begin{array}{c}\text { Tur } \\
\text { matrix } \\
\text { outer core }\end{array}$ & $\begin{array}{c}\text { Tur } \\
\text { matrix } \\
\operatorname{rim}\end{array}$ & $\begin{array}{c}\text { Tur } \\
\text { matrix } \\
\text { outer-rim }\end{array}$ & $\mathrm{Bt}$ & $\begin{array}{l}\text { Grt } \\
\text { core } \\
\end{array}$ & $\begin{array}{c}\text { Grt } \\
\text { mantle }\end{array}$ & $\begin{array}{l}\text { Grt } \\
\text { rim } \\
\end{array}$ & Phn \\
\hline$\overline{\mathrm{SiO}_{2}}$ & 33.33 & 34.26 & 32.00 & 33.30 & 34.63 & 34.5 & 33.94 & 34.13 & 35.2 & 50.5 \\
\hline $\mathrm{TiO}_{2}$ & 1.01 & 0.47 & 1.01 & 0.63 & 0.24 & 1.9 & n.d. & n.d. & n.d. & 0.41 \\
\hline $\mathrm{Al}_{2} \mathrm{O}_{3}$ & 26.43 & 29.8 & 25.01 & 28.74 & 28.71 & 15.4 & 19.26 & 19.56 & 20.2 & 21.2 \\
\hline $\mathrm{FeO}$ & 7.55 & 5.47 & 7.04 & 4.68 & 3.58 & 18.5 & 35.98 & 38.50 & 38.4 & 3.96 \\
\hline $\mathrm{MnO}$ & n.d. & n.d. & n.d. & n.d. & n.d. & 0.16 & 0.56 & 0.44 & 1.69 & 0.00 \\
\hline $\mathrm{MgO}$ & 7.14 & 7.23 & 6.82 & 7.12 & 8.62 & 10.3 & 3.11 & 3.22 & 2.85 & 4.66 \\
\hline $\mathrm{CaO}$ & 1.13 & 1.08 & 1.18 & 0.86 & 0.39 & n.d. & 2.95 & 1.04 & 0.56 & n.d. \\
\hline $\mathrm{Na}_{2} \mathrm{O}$ & 1.84 & 1.62 & 1.71 & 1.67 & 2.37 & 0.59 & n.d. & n.d. & n.d. & 0.23 \\
\hline $\mathrm{K}_{2} \mathrm{O}$ & n.d. & n.d. & n.d. & n.d. & n.d. & 9.04 & n.d. & n.d. & n.d. & 10.1 \\
\hline $\mathrm{BaO}$ & n.d. & n.d. & n.d. & n.d. & n.d. & n.d. & n.d. & n.d. & n.d. & n.d. \\
\hline Total & 78.43 & 79.94 & 74.78 & 77.00 & 78.54 & 90.4 & 95.80 & 96.89 & 98.80 & 91 \\
\hline Cation & & & & & & $\mathrm{O}=11$ & $\mathrm{O}=12$ & $\mathrm{O}=12$ & $\mathrm{O}=12$ & $\mathrm{O}=11$ \\
\hline $\mathrm{Si}$ & & & & & & 2.78 & 2.90 & 2.90 & 2.92 & 3.56 \\
\hline $\mathrm{Ti}$ & & & & & & 0.12 & - & - & - & 0.02 \\
\hline $\mathrm{Al}(\mathrm{IV})$ & & & & & & 1.22 & 0.10 & 0.10 & 0.08 & 0.44 \\
\hline $\mathrm{Al}(\mathrm{VI})$ & & & & & & 0.24 & 1.84 & 1.86 & 1.90 & 1.32 \\
\hline $\mathrm{Fe}^{\prime \prime \prime}$ & & & & & & - & - & - & - & - \\
\hline $\mathrm{Fe}^{\prime \prime}$ & & & & & & 1.25 & 2.57 & 2.73 & 2.67 & 0.23 \\
\hline $\mathrm{Mn}$ & & & & & & 0.01 & 0.04 & 0.03 & 0.12 & 0.00 \\
\hline $\mathrm{Mg}$ & & & & & & 1.24 & 0.40 & 0.41 & 0.35 & 0.49 \\
\hline $\mathrm{Ca}$ & & & & & & - & 0.27 & 0.09 & 0.05 & - \\
\hline $\mathrm{Na}$ & & & & & & 0.09 & - & - & - & 0.03 \\
\hline $\mathrm{K}$ & & & & & & 0.93 & - & - & - & 0.91 \\
\hline B & & & & & & - & - & - & - & - \\
\hline Total & & & & & & 7.88 & 8.13 & 8.12 & 8.09 & 7.01 \\
\hline
\end{tabular}

$\mathrm{Fe}^{\prime \prime \prime}$ of Cpx is calculated as per the method given in Banno (1959).

$\mathrm{Fe}^{\prime \prime \prime}$ of Amp is calculated when there are 5 cations at the $\mathrm{Y}$ site.

Incl, inclusion; f-matrix, fine-grained matrix.

1.0-2.5 GPa and $400-600{ }^{\circ} \mathrm{C}$ in the NKFMASH system (Wei and Powell, 2004). Consequently, glaucophane is probably a retrogressive phase in the quartz field, which is consistent with the absence of glaucophane in garnet.

\section{Occurrence of coesite and coesite-pseudomorph and K-Ar age}

Coesite and coesite-pseudomorph (Ps-coesite) appear widely in pelitic and quartz schists as an inclusion in garnet porphyroblast (see the locality of coesite and Pscoesite in Figs. 2 and 3). In contrast to pelitic schists, only two of 42 eclogite lenses among the 74 thin sections of eclogite checked bear the coesite-pseudomorph. Therefore, it is suggested that many quartz-bearing eclogites have not undergone UHPM, as is described later.

The $\mathrm{K}-\mathrm{Ar}$ age of phengite was determined from a coesite-pseudomorph bearing pelitic schists (Table 1).
Mineral separation was undertaken in the laboratory. Age determination was performed by Geospace Sciences Co. and Mass Spec Services Co. The K content was determined using flame photometry (Instrumental Laboratory Model 143). The isotopic ratio of Ar was determined by the isotopic dilution method using a mass spectrometer (Kratos MS-10S). The age calculation is based on the methods proposed by Dalrymple and Lanphere (1969) and Steiger and Jäger (1977). Two measurements were conducted for each sample, and consistent results were obtained. The $\mathrm{K}-\mathrm{Ar}$ age determination of UHP metamorphics is usually error-prone as Ar tends to be present in excess (e.g., Hacker and Wang, 1995). However, the $\mathrm{K}$-Ar phengite age for the UHP schist $(509 \pm 13 \mathrm{Ma})$ is consistent with the $\mathrm{K}-\mathrm{Ar}$ paragonite age of HP eclogite (482 \pm 17 Ma) (Tagiri et al., 1995) and the CHIME ages of monazites in UHP garnet-chloritoid-talc schists (481 \pm $26 \mathrm{Ma}$ and $480 \pm 56 \mathrm{Ma}$ ) (Togonbaeva et al., 2009), 
Table 3. (Continued 2)

\begin{tabular}{|c|c|c|c|c|c|c|c|c|c|c|c|c|c|c|c|c|c|}
\hline \multirow{3}{*}{$\begin{array}{l}\text { Sample } \\
\text { Mineral } \\
\text { Comment }\end{array}$} & \multicolumn{4}{|l|}{$34-53 \mathrm{~A}$} & \multicolumn{8}{|c|}{ 93062901B } & \multicolumn{5}{|c|}{$93070106 \mathrm{~A}$} \\
\hline & \multicolumn{4}{|l|}{ Eclogite } & \multicolumn{8}{|l|}{ Eclogite } & \multicolumn{5}{|c|}{ Eclogite } \\
\hline & $\begin{array}{c}\text { Omp } \\
\text { grt-incl }\end{array}$ & $\begin{array}{c}\text { Omp } \\
\text { rim }\end{array}$ & $\begin{array}{l}\text { Grt } \\
\text { core }\end{array}$ & $\begin{array}{l}\text { Grt } \\
\text { rim }\end{array}$ & $\begin{array}{c}\text { Omp } \\
\text { grt-incl }\end{array}$ & $\begin{array}{l}\text { Omp } \\
\text { core }\end{array}$ & $\begin{array}{l}\text { Grt } \\
\text { core }\end{array}$ & $\begin{array}{l}\text { Grt } \\
\text { rim }\end{array}$ & $\begin{array}{l}\text { Gln } \\
\text { core }\end{array}$ & $\begin{array}{c}\text { Amp } \\
\text { rim }\end{array}$ & $\begin{array}{l}\text { Amp } \\
\text { grt-incl }\end{array}$ & $\mathrm{Pg}$ & $\begin{array}{l}\text { Grt } \\
\text { rim }\end{array}$ & $\begin{array}{l}\text { Grt } \\
\text { core }\end{array}$ & $\begin{array}{l}\text { Gln } \\
\text { rim }\end{array}$ & $\begin{array}{l}\text { Amp } \\
\text { core }\end{array}$ & $\begin{array}{c}\text { Amp } \\
\text { rim }\end{array}$ \\
\hline$\overline{\mathrm{SiO}_{2}}$ & 55.58 & 56.1 & 37.30 & 38.4 & 55.73 & 55.91 & 37.54 & 37.54 & 55.78 & 47.30 & 38.80 & 46.70 & 38.25 & 37.1 & 57.5 & 53.4 & 50.9 \\
\hline $\mathrm{TiO}_{2}$ & 0.00 & 0.00 & 0.23 & 0.00 & 0.01 & 0.00 & 0.15 & 0.00 & 0.01 & 0.08 & 0.17 & 0.09 & 0.03 & 0.16 & 0.00 & 0.08 & 0.03 \\
\hline $\mathrm{Al}_{2} \mathrm{O}_{3}$ & 9 & 1 & 19.5 & 21 & 93 & 9.06 & 1985 & 9 & 11 & 8.64 & 12 & 38.70 & 2 & 4 & 9.61 & 52 & 4.70 \\
\hline $\mathrm{FeO}$ & & 4.34 & 26.4 & 2 & J & & & 28 & 28 & 14 & & 0.24 & $\mathrm{~J}$ & 30.9 & 13 & .7 & 17.9 \\
\hline $\operatorname{InO}$ & 0 & 0.00 & 2.89 & 0 & & 0. & 10 & & 1 & 7 & & 0.00 & 0.40 & 3.29 & 0.03 & .08 & 0.13 \\
\hline IgO & & 8 & 0.51 & 5.66 & & 7.90 & & & & & & 0.18 & & 0.44 & 9.4 & 2 & 12.4 \\
\hline $\mathrm{O}$ & & 1 & 12.8 & 5.84 & & 13 & & & & & & & & 8.66 & 0.69 & 40 & 9.92 \\
\hline $\mathrm{Na}_{2} \mathrm{O}$ & 41 & 8.04 & 0.03 & 0.00 & 9 & & & & & & & 8.13 & & 0.00 & 6.49 & .75 & 2.13 \\
\hline $\mathrm{K}_{2} \mathrm{O}$ & 0 & 0 & 0 & 0.00 & 0 & 0.00 & 0 & 0 & 03 & 7 & 0.00 & 0.20 & 0.00 & 0.00 & 0.00 & .07 & 0.00 \\
\hline Total & 99.99 & 100 & 99.7 & 99.9 & 99.90 & 100.22 & 99.36 & 100.22 & 96.84 & 95.63 & 96.44 & 94.39 & 99.77 & 101 & 96.7 & 97.2 & 98.1 \\
\hline Cation & $\mathrm{O}=6$ & $\mathrm{O}=6$ & $\mathrm{O}=12$ & $\mathrm{O}=12$ & $\mathrm{O}=6$ & $\mathrm{O}=6$ & $\mathrm{O}=12$ & $\mathrm{O}=12$ & $\mathrm{O}=23$ & $\mathrm{O}=23$ & $\mathrm{O}=23$ & $\mathrm{O}=11$ & $\mathrm{O}=12$ & $\mathrm{O}=12$ & $\mathrm{O}=23$ & $\mathrm{O}=23$ & $\mathrm{O}=23$ \\
\hline $\mathrm{Si}$ & 2.02 & 1.99 & 3.00 & 3.01 & 2.02 & 2.01 & 3.04 & 2.98 & 7.74 & 7.01 & 5.94 & 3.00 & 3.05 & 2.97 & 7.98 & 7.65 & 7.35 \\
\hline $\mathrm{Ti}$ & 00 & 0.00 & 0.01 & 0.00 & 0.00 & 0.00 & 0.01 & 0.00 & 0.00 & 0.01 & 0.02 & 0.00 & 0.00 & 0.01 & 0.00 & 0.01 & 0.00 \\
\hline (IV) & & 0 & 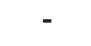 & - & - & - & - & & & & & 1.00 & - & 0.03 & 0.02 & 0.35 & 0.65 \\
\hline (VI) & & 5 & 0 & 1.9 & & & & & & & & 1.93 & & 1.90 & 1.55 & 0.41 & 0.15 \\
\hline $\mathrm{Fe}^{\prime \prime \prime}$ & 21 & 0.10 & 0.15 & 0.04 & 16 & 13 & 0.11 & 0.02 & 0.21 & 0.43 & 0.99 & 0.09 & 0.00 & 0.10 & 0.52 & 0.57 & 0.83 \\
\hline $\mathrm{Fe}^{\prime \prime}$ & 4 & 0.03 & 1.62 & 1.84 & 4 & 5 & 1. & & 86 & 3 & 1.72 & 0.00 & 09 & 1.97 & 0.98 & 1.19 & 1.33 \\
\hline 111 & & 0.00 & 0.20 & 0.01 & & 0 & & & 0.02 & 0.02 & 0.42 & 0.00 & 0.03 & 0.22 & 0.00 & 0.01 & 0.02 \\
\hline & & 0.44 & .06 & 0.66 & & & & & & 2.69 & & 0.02 & & 0.05 & 1.94 & 2.81 & 2.68 \\
\hline $\mathrm{Ca}$ & 6 & 0.48 & 1.10 & 0.49 & 1 & 52 & 0. & 0.67 & 0.29 & 1.48 & 1.59 & 0.01 & 0.47 & 0.74 & 0.10 & 1.29 & 1.54 \\
\hline $\mathrm{Na}$ & & 0.55 & 0.01 & 0.00 & & & 0.00 & & 1.93 & 1.03 & 0.78 & 1.01 & & 0.00 & 1.90 & 0.77 & 0.61 \\
\hline & & & & 0 & & & & & 0.01 & 0.03 & 0.00 & 0.02 & & 0.00 & 0.00 & 0.01 & 0.00 \\
\hline Total & 4.09 & 4.05 & 8.00 & 8.00 & 4.07 & 4.05 & 8.00 & 8.00 & 15.22 & 15.54 & 15.37 & 7.08 & 8.00 & 8.00 & 15.00 & 15.08 & 15.14 \\
\hline
\end{tabular}

$\mathrm{Fe}^{\prime \prime \prime}$ of Cpx is calculated as per the method given in Banno (1959). $\mathrm{Fe}^{\prime \prime \prime}$ of Amp is calculated when there are 5 cations at the $\mathrm{Y}$ site.

Incl, inclusion; f-matrix, fine-grained matrix.

which indicates that the $\mathrm{K}-\mathrm{Ar}$ ages can be used in the discussion of the metamorphic event. These $\mathrm{K}-\mathrm{Ar}$ ages pertain to the later stage of metamorphism, i.e., after the UHPM. These ages of $\sim 500 \mathrm{Ma}$ are comparable with the UHPM ages of the Kokchetav and of the North Qaidam (Katayama et al., 2001; Yang et al., 2001; Rumble et al., 2005).

\section{DIFFERENT GROWTH ZONINGS OF MINERALS IN ECLOGITES}

\section{Zonal structure of garnets in eclogites}

Electron probe microanalysis (EPMA) and energy dispersive spectroscopy-scanning electron microscopy (EDSSEM) were performed at Ibaraki (JCXA-733 and JSM5600LV), Shimane, and Yamagata Universities (JCXA8600 ) to analyze the compositions, zonal structure, and compositional mapping of the minerals. An accelerating voltage of $15 \mathrm{kV}$ was used for all equipment, and the cor- rection method of Bence and Albee (1968) was used.

49 grains of garnet in eight thin section samples of different eclogite lenses were analyzed. Some data from Puelles and Takasu (1996) are also cited. Garnet grains that were cut through their core were carefully selected. The zonal structure of garnet in eclogites is divided into three types as follows (Table 3 and Figs. 8 and 9):

$$
\begin{aligned}
\text { Type I: } & \text { Core } \\
& \left(\operatorname{Alm}_{33-52} \operatorname{Sps}_{14-27} \operatorname{Pyr}_{1-4} \mathrm{Grs}_{28-51}\right) \\
& - \text { Mantle }\left(\mathrm{Alm}_{46-67} \mathrm{Sps}_{2-21} \mathrm{Pyr}_{2-11} \mathrm{Grs}_{21-30}\right) \\
& -\operatorname{Rim}\left(\mathrm{Alm}_{53-66} \mathrm{Sps}_{2-6} \mathrm{Pyr}_{8-12} \mathrm{Grs}_{22-34}\right),
\end{aligned}
$$

Type II: Core ( $\left.\mathrm{Alm}_{57-75} \mathrm{Sps}_{4-8} \mathrm{Pyr}_{2-7} \mathrm{Grs}_{15-35}\right)$

- Mantle $\left(\mathrm{Alm}_{69-77} \mathrm{Sps}_{0-7} \mathrm{Pyr}_{4-10} \mathrm{Grs}_{14-22}\right)$

- Rim $\left(\mathrm{Alm}_{59-74} \mathrm{Sps}_{0-0.6} \mathrm{Pyr}_{10-24} \mathrm{Grs}_{12-17}\right)$,

Type III: Core ( $\left.\mathrm{Alm}_{60-73} \mathrm{Sps}_{2-7} \mathrm{Pyr}_{2-4} \mathrm{Grs}_{20-35}\right)$

- Mantle ( $\left.\mathrm{Alm}_{68-73} \mathrm{Sps}_{1-2} \mathrm{Pyr}_{3-8} \mathrm{Grs}_{21-26}\right)$

- Rim $\left(\mathrm{Alm}_{70-78} \mathrm{Sps}_{0-1} \mathrm{Pyr}_{4-10} \mathrm{Grs}_{14-22}\right)$. 
Table 3. (Continued 3)

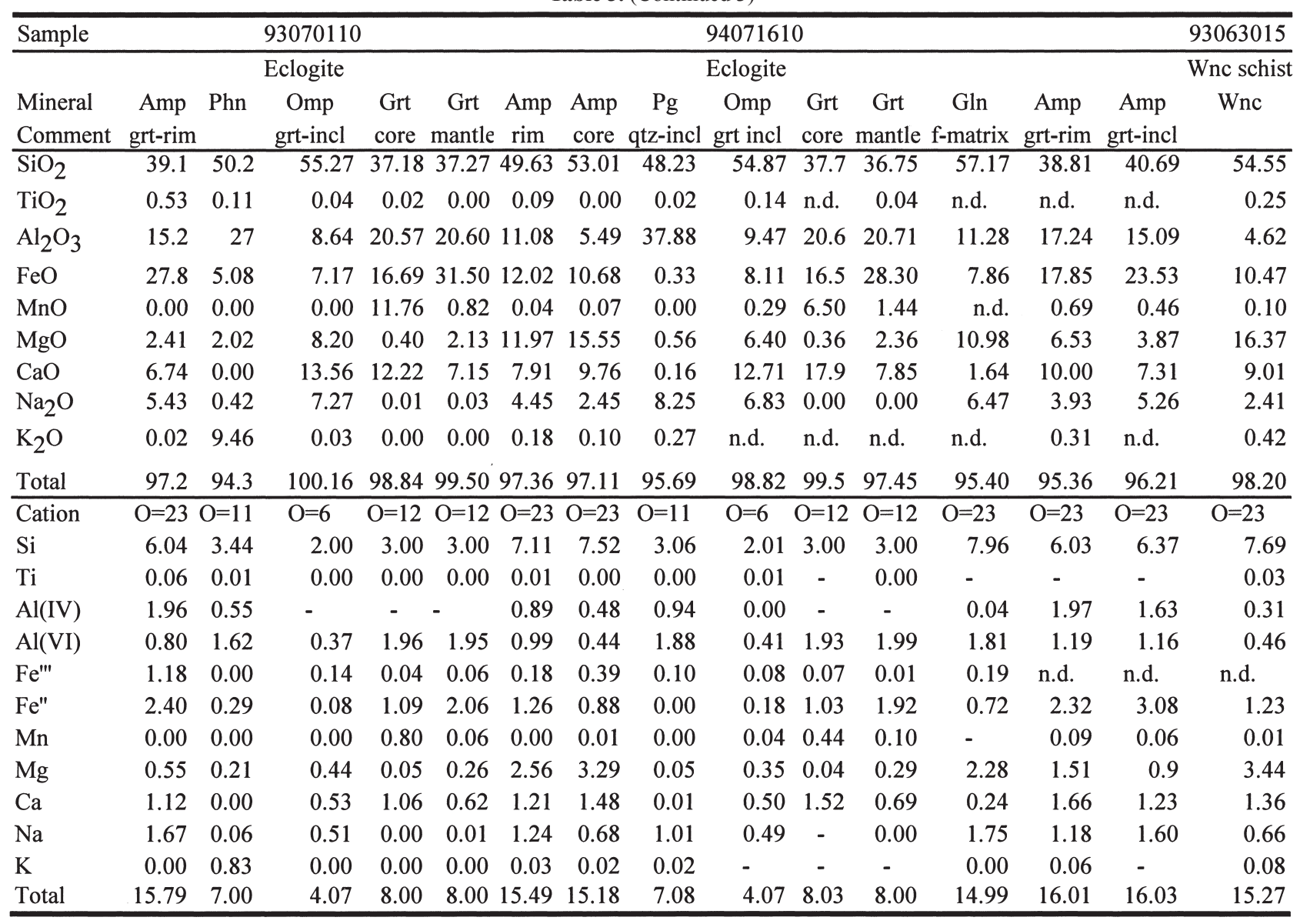

$\mathrm{Fe}^{\prime \prime \prime}$ of Cpx is calculated as per the method given in Banno (1959).

$\mathrm{Fe}^{\prime \prime \prime}$ of Amp is calculated when there are 5 cations at the Y site.

Incl, inclusion; f-matrix, fine-grained matrix.

The mantle of type I garnet is divided into two subzones. In type I garnet, the Ca content decreases from the core to the mantle with the wide compositional gap, and the $\mathrm{Mn}$ content distinctly decreases from mantle I to mantle II (Fig. 9). The Fe content of type I garnet increases from the core to mantle II through mantle I as decreasing in $\mathrm{Ca}$ and $\mathrm{Mn}$. The rim of type I garnet is slightly rich in $\mathrm{Mn}$ and $\mathrm{Mg}$ as compared to the mantle. The compositional variation of type II is continuous from core to rim and rich in $\mathrm{Mg}$ at the outermost rim. Figures $10 \mathrm{a}-10 \mathrm{~h}$ show the compositional mapping of type I and type II garnet. Types I and II have a prograde zonal structure from the core to the rim. The zoning of type III is not conspicuous; in other words, it is homogeneous. Garnet in an eclogite can have one of three types of zonal structure.

All types of garnets are associated with omphacite. We estimated the ferric iron content in omphacite using two methods (Table 4). The maximum difference in temperature between the two methods reaches $100{ }^{\circ} \mathrm{C}$, but the differences in the estimated temperatures are mostly with- in $50{ }^{\circ} \mathrm{C}$. The $\mathrm{K}_{\mathrm{D}}(\mathrm{Fe} / \mathrm{Mg})$ between garnet and the omphacite inclusion in the garnet ranges from $\mathrm{K}_{\mathrm{D}}=108$ to $\mathrm{K}_{\mathrm{D}}=$ 21 (Table 4 and Fig. 11). The garnet sample 93062901B contains omphacite in its mantle and rim. The garnet sample 94071610 contains omphacite in its core and mantle. The other three garnet samples contain omphacite in their mantles. The high content of $\mathrm{Ca}$ in garnet sample 94071610 gives the lower temperature in the calculation proposed by Krogh (1988) as indicated by Hirajima and Nakamura (2003).

The eclogite facies mineral assemblages of eclogite contain a limited number of phases such as (Grt + Omp + $\mathrm{Ep} \pm \mathrm{Pg} \pm$ Amp) (Table 2). This assemblage may be dealt with in terms of a five-component system, $\mathrm{Al}_{2} \mathrm{O}_{3}-\mathrm{FeO}-$ MgO-CaO- $\mathrm{Na}_{2} \mathrm{O}$, with an excess of $\mathrm{SiO}_{2}$ and $\mathrm{H}_{2} \mathrm{O}$ (e.g., Hirajima et al., 1988). In three eclogites, taramite, barroisite, and glaucophane occur as an inclusion in garnet associated with omphacite (Table 2). When we consider the occurrence of several phases of amphiboles, the number of degree of freedom of this system becomes more 

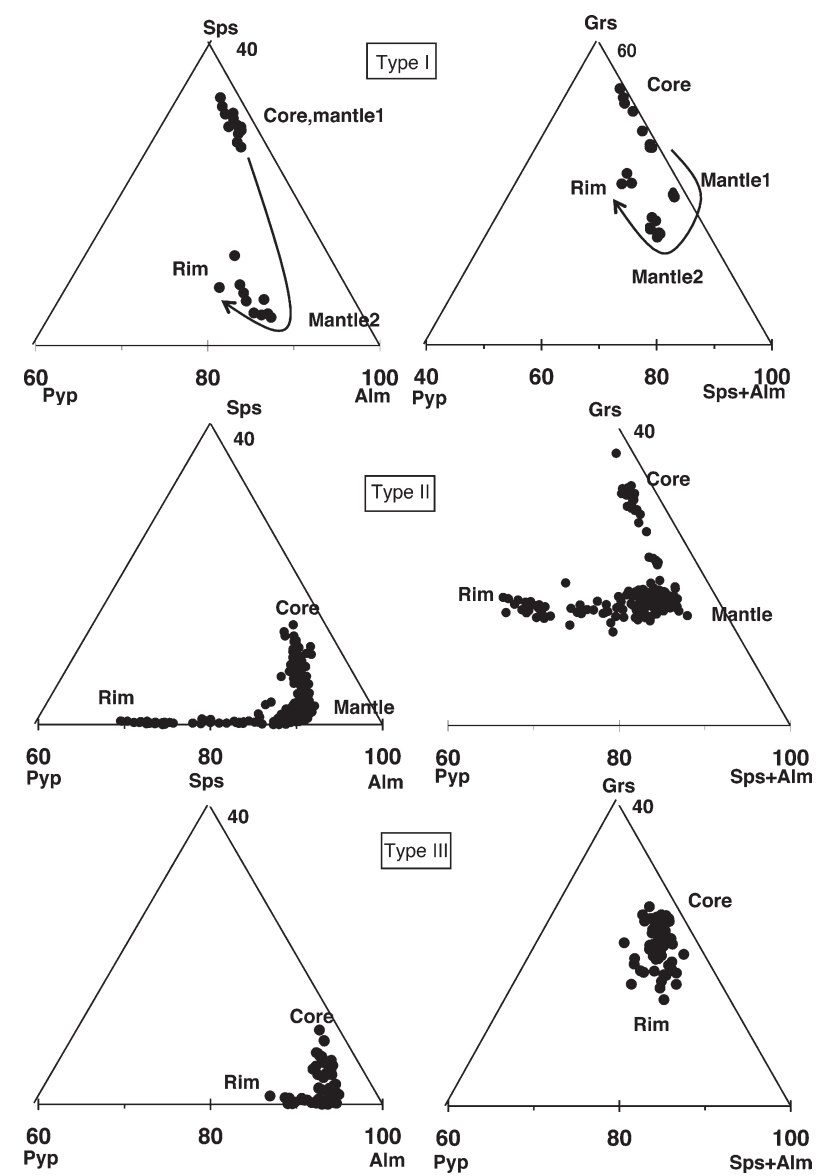

Figure 8. Compositional zoning of the three types of garnet in eclogites.

than three degrees and consequently the different compositional zoning of garnet is controlled by several factors such as $P, T$, and bulk chemical composition. On the other hand, Grt-Omp geothermometer calculations for the garnet mantle at the peak pressure condition of $2 \mathrm{GPa}$ gives different physical conditions for type I garnet (375-662 ${ }^{\circ} \mathrm{C}$ or $\left.388-582{ }^{\circ} \mathrm{C}\right)$, type II garnet $\left(293-438{ }^{\circ} \mathrm{C}\right.$ or $\left.369-403{ }^{\circ} \mathrm{C}\right)$, and type III garnet $\left(326-420^{\circ} \mathrm{C}\right.$ or $397-513$ $\left.{ }^{\circ} \mathrm{C}\right)$ (Table 4). Type I and type II garnets are of the lowtemperature UHPM and HPM. Moreover, the mantle of type I garnets of three eclogites underwent crystallization at different temperatures (Table 4): sample 93062901B, $375-405{ }^{\circ} \mathrm{C}$ (or $388-401{ }^{\circ} \mathrm{C}$ ); sample $93070110,412-510$ ${ }^{\circ} \mathrm{C}$ (or $404-461{ }^{\circ} \mathrm{C}$ ); and sample $94071610,563-662{ }^{\circ} \mathrm{C}$ (or $543-582{ }^{\circ} \mathrm{C}$ ). This variation also indicates that the eclogites recrystallized at different temperatures under certain pressure conditions (Ellis and Green, 1979; Powell, 1985) (Table 4). The very rare appearance of coesitepseudomorph in eclogites (for example, only in two out of 74 thin sections of 42 eclogite lenses) is consistent with the above consideration.
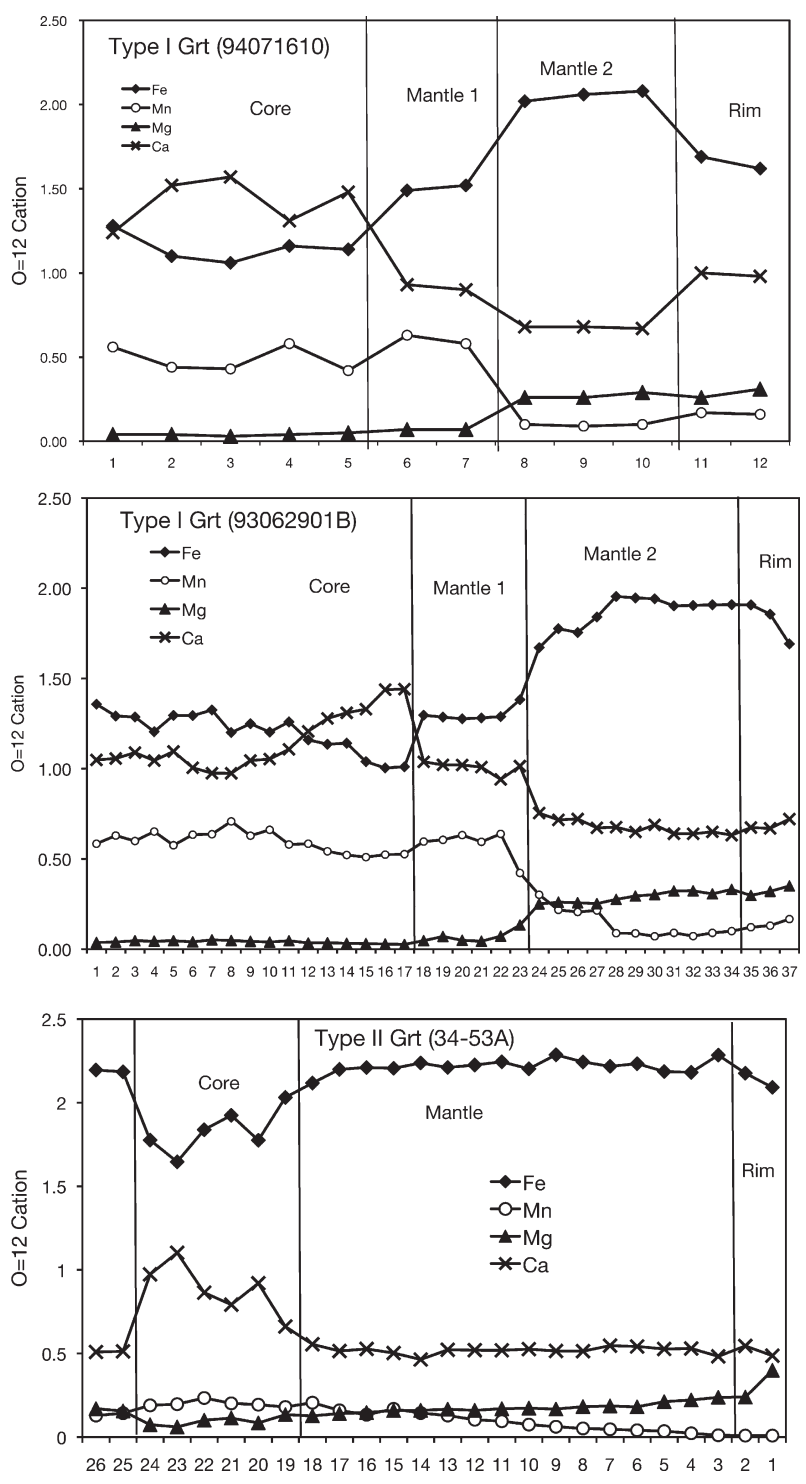

Figure 9. Compositional profiles of type I and type II garnets.

\section{Zonation of Na-Ca amphiboles in eclogites}

Porphyroblastic $\mathrm{Na}-\mathrm{Ca}$ amphiboles and sodic actinolite show various compositional zonations. Barroisite, taramite, and glaucophane inclusions in garnet, and the barroisite and glaucophane core of porphyroblastic $\mathrm{Na}-\mathrm{Ca}$ amphiboles in eclogite are associated with omphacite and garnet by direct contact. The mantle and rim of $\mathrm{Na}-\mathrm{Ca}$ amphiboles do not show eclogite facies metamorphism because they do not have direct contact with omphacite. The mantle and rim of $\mathrm{Na}-\mathrm{Ca}$ amphiboles probably crystallized at the glaucophane schist facies or the epidote amphibolite facies. As one example, in sample 34-53A, amphiboles vary from the barroisite core to the sodic actinolite rim with fine-grained glaucophane in the matrix (Fig. 12a). In sample 93062901B, porphyroblastic amphi- 

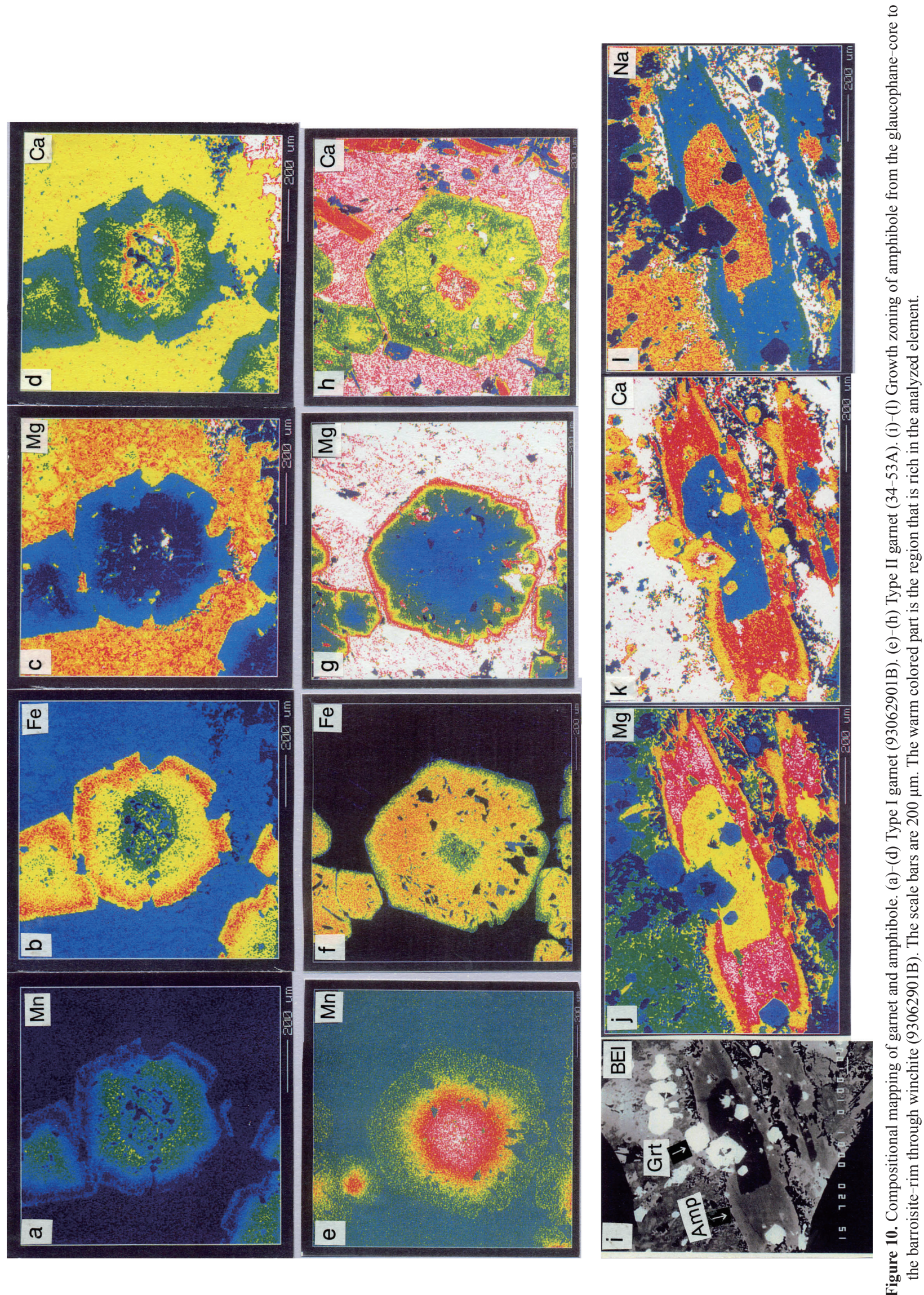
Table 4. Temperature estimation at 2 GPa using the Grt-Omp geothermometer ${ }^{*}$

\begin{tabular}{|c|c|c|c|c|c|c|c|c|c|c|c|c|c|c|c|c|}
\hline \multirow{2}{*}{$\begin{array}{c}\text { 82-2641 } \\
\text { Cpx- } \\
\text { incl }\end{array}$} & \multirow[b]{2}{*}{ Cpx2 } & \multicolumn{3}{|c|}{ Type III } & \multicolumn{7}{|c|}{ 93062901B } & \multicolumn{2}{|c|}{ Type I } & \multirow[b]{2}{*}{$\operatorname{Grt}(\mathrm{Ca})$} & \multirow[b]{2}{*}{ Temp } & \\
\hline & & XJd & $\begin{array}{c}\text { Grt- } \\
\text { mant }\end{array}$ & $\mathrm{KD}$ & $\mathrm{Grt}(\mathrm{Ca})$ & Temp & Temp2 & Zone & $\begin{array}{l}\text { Cpx- } \\
\text { incl }\end{array}$ & Cpx2 & XJd & Grt & $\mathrm{KD}$ & & & \\
\hline 0.15 & 0.22 & 0.44 & 8.58 & 57.18 & 0.30 & 405 & 453 & mantle & 0.16 & 0.20 & 0.39 & 19.50 & 125.81 & 0.40 & 375 & $\overline{401}$ \\
\hline 0.16 & 0.31 & 0.45 & 5.77 & 37.23 & 0.21 & 411 & 513 & mantle & 0.20 & 0.21 & 0.38 & 18.90 & 94.50 & 0.36 & 383 & 388 \\
\hline 0.15 & 0.17 & 0.34 & 8.11 & 53.36 & 0.31 & 420 & 434 & mantle & 0.36 & 0.31 & 0.37 & 24.30 & 68.07 & 0.33 & 405 & 389 \\
\hline 0.08 & 0.15 & 0.38 & 8.25 & 108.55 & 0.27 & 326 & 397 & rim & 0.24 & 0.38 & 0.30 & 6.78 & 28.61 & 0.32 & 512 & 592 \\
\hline 9307011 & & & Type I & & & & & $34-53 \mathrm{~A}$ & & & Type II & & & & & \\
\hline $\begin{array}{c}\text { Cpx- } \\
\text { incl }\end{array}$ & Cpx2 & XJd & $\begin{array}{c}\text { Grt- } \\
\text { mant }\end{array}$ & $\mathrm{KD}$ & Grt(Ca) & Temp & Temp2 & Cpx-incl & Cpx2 & $\mathrm{Xjd}$ & $\begin{array}{l}\text { Grt- } \\
\text { mant }\end{array}$ & $\mathrm{KD}$ & $\mathrm{Grt}(\mathrm{Ca})$ & Temp & Temp2 & \\
\hline 0.14 & 0.13 & 0.44 & 5.85 & 41.20 & 0.24 & 415 & 404 & 0.08 & 0.17 & 0.38 & 6.24 & 75.18 & 0.14 & 293 & 369 & \\
\hline 0.17 & 0.23 & 0.39 & 7.39 & 43.47 & 0.25 & 412 & 452 & 0.09 & 0.19 & 0.42 & 6.51 & 74.06 & 0.15 & 297 & 381 & \\
\hline 0.19 & 0.14 & 0.43 & 4.51 & 23.86 & 0.27 & 506 & 461 & 0.32 & 0.25 & 0.34 & 7.86 & 24.33 & 0.16 & 438 & 403 & \\
\hline 0.28 & 0.15 & 0.46 & 5.93 & 21.18 & 0.24 & 510 & 420 & & & & & & & & & \\
\hline 9407161 & & & & Type I & & & & & & & & & & & & \\
\hline Zone & $\begin{array}{l}\text { Cpx- } \\
\text { incl }\end{array}$ & Cpx2 & XJd & Grt & $\mathrm{KD}$ & $\mathrm{Grt}(\mathrm{Ca})$ & Temp & Temp2 & by Krog & $h(1988)$ & & & & & & \\
\hline core & 0.50 & 0.40 & 0.41 & 34.70 & 69.40 & 0.52 & 501 & 473 & 421 & & & & & & & \\
\hline mantle & 0.76 & 0.67 & 0.37 & 24.40 & 32.02 & 0.43 & 563 & 543 & 515 & & & & & & & \\
\hline mantle & 1.04 & 0.66 & 0.43 & 24.30 & 23.37 & 0.50 & 662 & 582 & 585 & & & & & & & \\
\hline
\end{tabular}

* Powell, 1985 and Krogh, 1988.

$\mathrm{Cpx}$-incl, $\mathrm{Fe}^{\prime \prime} / \mathrm{Mg}$ ratio of clinopyroxene inclusion; Grt-core, Fe"/Mg ratio of garnet core; Grt-mant, Fe"/Mg ratio of garnet mantle; $\mathrm{Grt}(\mathrm{Ca})$, grossular molecule of garnet mantle; XJd, jadeite mol ratio in omphacite.

Cpx2 and Temp2 are determined using the following method for calculating the ferric iron content in omphacite. The ferric iron content in omphacite is estimated on the basis of maximum fitting to the clinopyroxene structural formula when we used an appropriate $\mathrm{Fe}^{3+} / \mathrm{Fe}^{2+} \mathrm{ratio}_{\text {. }}$

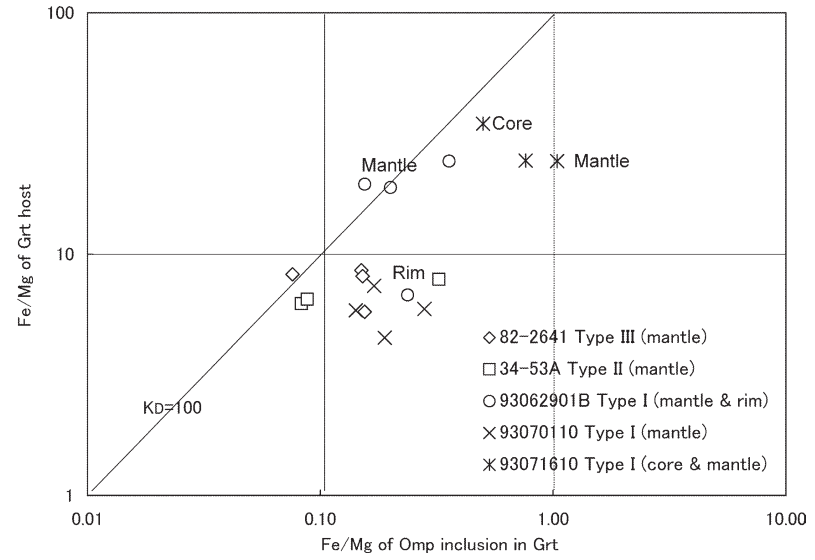

Figure 11. $\mathrm{K}_{\mathrm{D}}(\mathrm{Fe} / \mathrm{Mg})$ between garnet and omphacite inclusion in the garnet.

boles vary from the glaucophane core to the sodic actinolite rim through the barroisite and taramite mantle $(\mathrm{Si}=$ 5.75) (Fig. 12c). Figures 10i-101 indicate the compositional map of $\mathrm{Na}-\mathrm{Ca}$ amphiboles in the eclogite of Figure 12c. In sample 94071601, porphyroblastic amphiboles change from the glaucophanitic core to the barroisitic rim through the winchite mantle (Fig. 12d). Moreover, in sample 93070106A, porphyroblastic amphiboles change from the winchite core and mantle to the glaucophanitic rim (Fig. 12e). In contrast to these samples, amphiboles of eclogite (93070110) (Fig. 12b) have a narrow variation from the sodic actinolite core to the barroisite rim. As shown in Figure 13, there is various retrograde-growth zoning of $\mathrm{Na}-\mathrm{Ca}$ amphiboles except the core, and they differ for every sample. As a result, we cannot relate the zonation path of $\mathrm{Na}-\mathrm{Ca}$ amphiboles to the three types of zoned garnets.

Amphibole zonation in metabasites records the evolution of metamorphic conditions (e.g., Brown, 1977). Holland and Richardson (1979) indicated the $P-T$ path in terms of the glaucophane activity and the edenite activity of amphibole zonation. These activities depend on the interactions with excess components of albite, clinozoisite, chlorite, quartz, and vapor in the epidote-amphibolite facies or the glaucophane-schist facies. These excess component minerals, except for chlorite, always occur as a retrograde phase in the present eclogite lenses. According to Holland and Richardson (1979), glaucophane is dominant at high $P$ and low $T$, edenite is dominant at low $P$ and high $T$, and tremolite is dominant at low $P$ and low $T$. In other words, barroisite enrichment occurs under medium $P$ and $T$ conditions, glaucophane enrichment occurs under high $P$ and low $T$ conditions, and actinolitewinchite enrichment occurs under medium $P$ and low $T$ conditions. Therefore, the zonation of the mantle and that of the rim of $\mathrm{Na}-\mathrm{Ca}$ amphiboles indicate that each of the present eclogite lenses has a different $P-T$ history in the ascending stage. 
$\mathrm{Na}(\mathrm{B})$

(a) 34-53A Eclogite

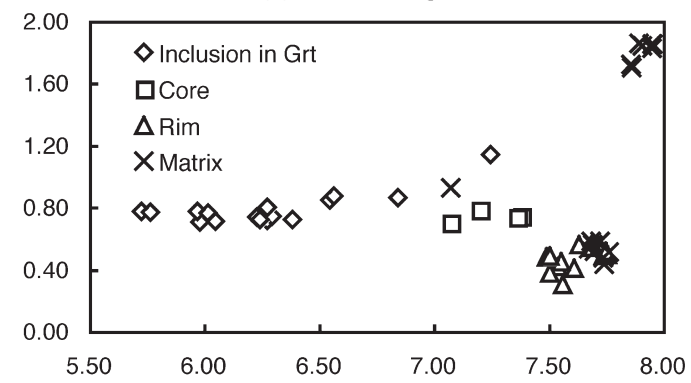

(c) $93062901 \mathrm{~B}$ Eclogite

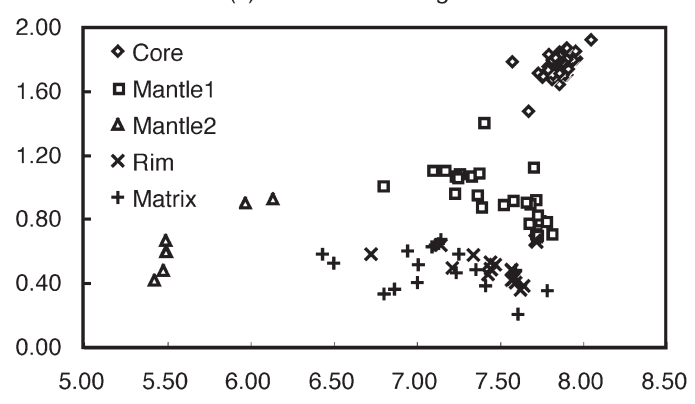

(e) $93070106 \mathrm{~A}$ Eclogite

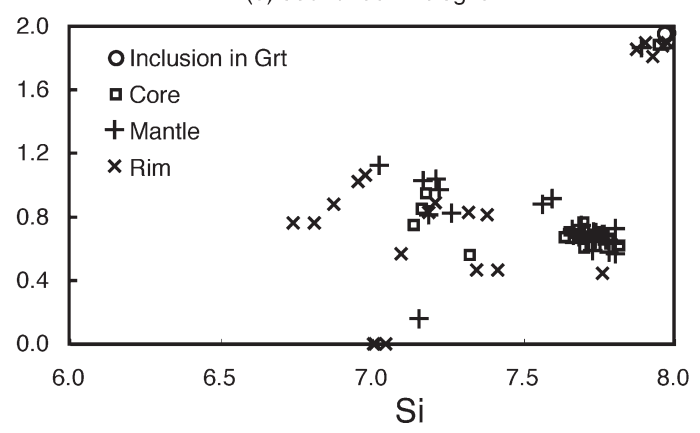

$\mathrm{Na}(\mathrm{B}) \quad$ (b) 93070110 Eclogite

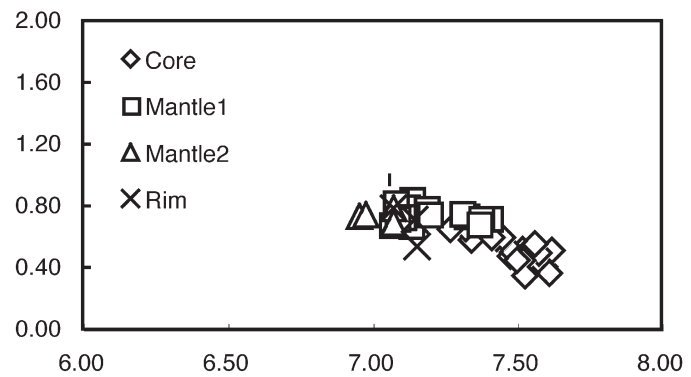

(d) Eclogites

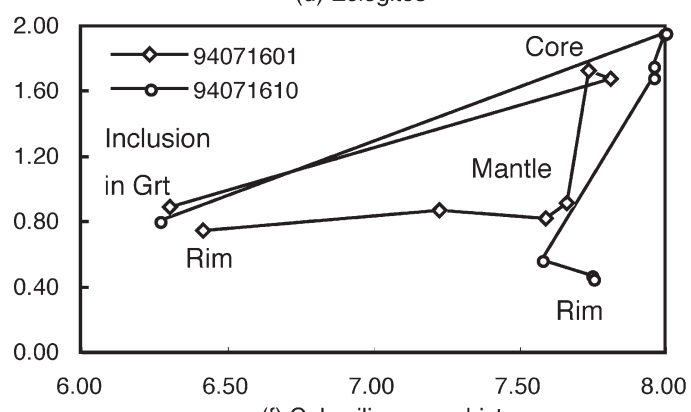

(f) Calc-siliceous schist

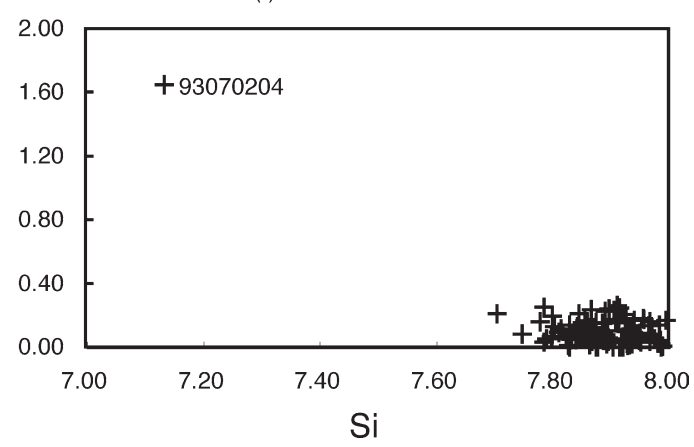

Figure 12. Zonation of amphibole inclusions in garnet and of porphyroblastic crystals in eclogites. Fine-grained amphiboles dispersed in the matrix of eclogites and actinolite of calc-siliceous schist are also shown in the figure.

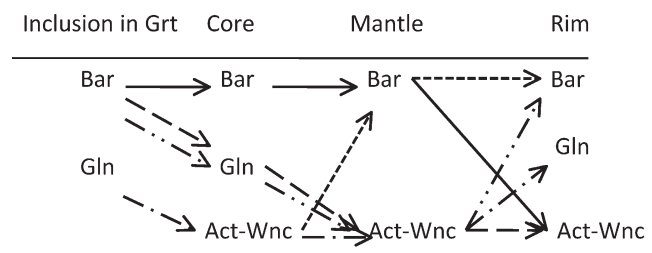

Contrast to the three types of garnet

Type I $-\rightarrow 94071610$ Type II $\longrightarrow$ 34-53A Type III $-\rightarrow$ 93070106A

$$
\rightarrow--\rightarrow 93070110
$$$$
-\cdots \rightarrow 94071601
$$

Figure 13. Summary of the zonation of $\mathrm{Na}-\mathrm{Ca}$ amphiboles in eclogites without the zonation of fine-grained amphiboles in the matrix of eclogites. The associated relation between the three types of garnet and the zonation of $\mathrm{Na}-\mathrm{Ca}$ amphiboles is indicated by the different arrows.

Applying geothermobarometry to the $\mathrm{Na}-\mathrm{Ca}$ amphibole mantle of the present samples according to the method proposed by Holland and Richardson (1979), a retrograde $P-T$ path of eclogites can be calculated. Sample 93062901B experienced $\sim 1.3 \mathrm{GPa}$ at $\sim 300{ }^{\circ} \mathrm{C}$ during the retrograde metamorphism and reached approximately 0.8 $\mathrm{GPa}$ at $\sim 400{ }^{\circ} \mathrm{C}$. The retrograde $P-T$ conditions of samples 34-53A, 93070106A, and 93070110 reached approximately $0.8 \mathrm{GPa}$ at $\sim 500-300{ }^{\circ} \mathrm{C}$ (Fig. 14). The metamorphic age of one of the typical eclogites, viz., 82-2641, is the same as that of the host pelitic schists. Thus, many eclogite lenses are cognate xenoliths in the tectonic mélange.

Symplectic breakdown products from omphacite typically include calcic clinopyroxene or calcic amphiboles. Actinolite and hornblende rarely occur in the matrix of eclogites as the product of the latest metamorphism of amphibolitization.

\section{EXOTIC BLOCKS IN THE UHPM ROCKS}

\section{Calc-siliceous schist lens}

Carbonaceous schists are commonly intercalated with 


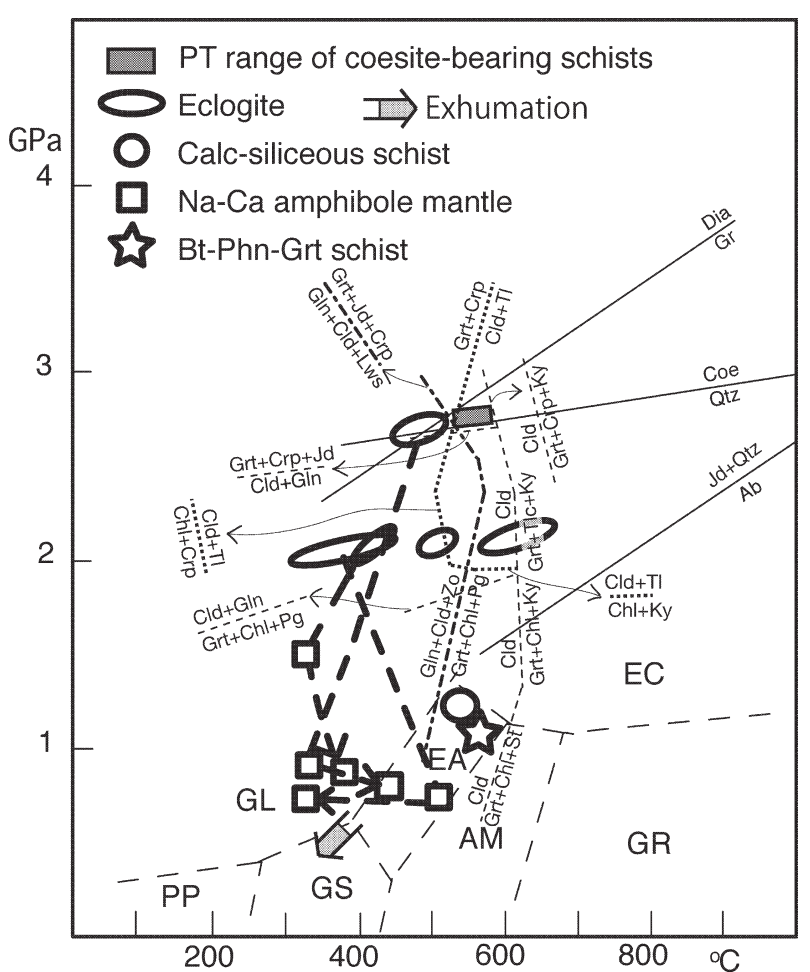

Figure 14. $P-T$ estimation of the Makbal Complex. The maximum stability field of chloritoid is quoted from Wei and Powell (2004), the graphite-diamond transition curve from Kennedy and Kennedy (1976), the quartz-coesite transition line from Mirwald and Massonne (1980), the albite $=$ jadeite + quartz from Holland (1980). The broken lines show the maximum stability limits of chloritoid and the stability field of glaucophane + chloritoid in the NKFMASH (Wei and Song, 2008). The dotted broken lines show the stability limit of glaucophane + chloritoid in NCKFMASH (Wei and Song, 2008). The dotted lines show the stability field of chloritoid + talc in KFMASH (Wei and Powell, 2003). PP, prehnite-pumpellyite facies; GS, greenschist facies; GL, glaucophane schist facies; EA, epidote amphibolite facies; AMP, amphibolite facies; GRAN, granulite facies; ECL, eclogite facies.

UHP pelitic schists of the Makbal Complex. A small lens of calc-siliceous schist, which is approximately $3 \mathrm{~m}$ long and $1 \mathrm{~m}$ thick, is included in these schists (see Fig. 2). Albite, diopside, actinolite, phengite, phlogopite, K-feldspar, epidote, and quartz are associated in the calc-siliceous schist lens. Table 3 lists the chemical compositions of the constituent minerals (Bakirov et al., 1998) of the calc-siliceous schist lens. Actinolite and diopside are slightly sodic, $\mathrm{K}$-feldspar contains small amount of $\mathrm{Ba}$, and albite forms a porphyroblast (Fig. 5e). This assemblage is of the epidote amphibolite facies. There is no relic evidence of UHP conditions in this lens. The phengite geobarometer, which is based on the limiting assemblage of phengite + $\mathrm{K}$-feldspar + quartz (Massonne and Schreyer, 1987), yields only 1.1 to $1.3 \mathrm{GPa}$ at $600{ }^{\circ} \mathrm{C}$, i.e., at the maximum temperature of this UHPM complex (cf., Bakirov et al.,
Table 5. $P-T$ estimation using the plagioclase-biotite-garnet-muscovite geothermobarometer ${ }^{*}$ for a biotite-phengite-garnet schist

\begin{tabular}{|c|c|c|c|c|c|c|c|}
\hline $\begin{array}{l}\text { Grt- } \\
\text { Zone }\end{array}$ & $\begin{array}{l}\text { Ks } \\
(1)\end{array}$ & $\begin{array}{l}\mathrm{Ks} \\
(2)\end{array}$ & $\begin{array}{c}\operatorname{lnKs} \\
(1)\end{array}$ & $\begin{array}{c}\ln K s \\
(2)\end{array}$ & $\begin{array}{c}\operatorname{lnKs} \\
(3)\end{array}$ & $\begin{array}{c}\mathrm{T}^{\circ} \mathrm{C} \\
(\mathrm{K} 1-\mathrm{K} 3)\end{array}$ & P kbar \\
\hline rim & 3240 & 7.635 & 8.083 & 2.033 & -6.05 & 495 & 5.4 \\
\hline mantle & 395.6 & 1.357 & 5.980 & 0.305 & -5.68 & 536 & 7.3 \\
\hline core & 19.58 & 0.074 & 2.974 & -2.6 & -5.58 & 557 & 10 \\
\hline
\end{tabular}

${ }^{*}$ Ghent and Stout, 1981.

$\mathrm{Ks}(1), \mathrm{Ks}(2)$, and $\mathrm{Ks}(3)$ are calculated using the equation proposed by Ghent and Stout (1981).

The compositions of the associated minerals are also shown in the Table 3 .

1998) (Fig. 14). Therefore, the calc-siliceous schist lens has not experienced UHPM.

\section{Biotite-phengite-garnet schist lens}

A fine-grained biotite-phengite-garnet schist lens is included in the UHPM rocks (Fig. 3). The lens is $\sim 3 \mathrm{~m}$ thick and $\sim 10 \mathrm{~m}$ long (Fig. $4 \mathrm{f}$ ). In this schist, biotite is stable and associated with garnet, phengite, and albite (Fig. $5 \mathrm{f}$ and Table 2), in contrast to the instability of biotite in the host UHP pelitic schist. There is no relic evidence of UHP conditions in this lens. The plagioclase-biotite-garnet-muscovite geothermobarometer (Ghent and Stout, 1981) of this schist yields $488-557^{\circ} \mathrm{C}$ and $0.54-1$ GPa (Table 5). The K-Ar ages of biotite and phengite are $769 \pm 19$ and $717 \pm 18 \mathrm{Ma}$, respectively (Table 1). The two ages are relatively consistent with each other and effective for discussion, even if the $\mathrm{K}-\mathrm{Ar}$ age dating system is error-prone because of the excess Ar. As a result, the biotite-phengite-garnet schist is not an UHPM rock, and its metamorphism is not the same as the Makbal UHPM.

\section{Winchite schist lens}

A small lens of winchite schist occurs in the UHPM rocks (Fig. 3). The constituent minerals consist of a large amount of winchite and a small amount of albite (Table 2). Winchite is homogeneous in composition and does not show any growth zoning (Table 3). The winchite schist does not have any relic evidence of UHPM. K-Ar age dating of winchite gives $881 \mathrm{Ma}$. The $\mathrm{K}$ and Ar contents of this sample are very low and therefore the result contains significant error, but the age is clearly different from the Makbal UHPM age.

In conclusion, a calc-siliceous schist lens, biotitephengite-garnet schist, and winchite schist are exotic blocks in the tectonic mélange. These exotic blocks were 
situated at a shallow depth in the stable field of quartz and albite.

\section{DISCUSSION}

Figure 14 shows the $P-T$ estimation of the Makbal Complex. The metamorphic condition of the UHP host rocks reaches approximately $2.8 \mathrm{GPa}$ at $\sim 560{ }^{\circ} \mathrm{C}$, and then ascends adiabatically in the stable field of chloritoid + talc. The $P-T$ path of the UHP host rocks shifts to the lower temperature side due to the change in the stability of chloritoid + talc caused by the $\mathrm{P}_{\mathrm{H}_{2} \mathrm{O}}$ and $\mathrm{Mg} / \mathrm{Fe}$ variation of the system. $P-T$ estimation of the mantle of $\mathrm{Na}-\mathrm{Ca}$ amphiboles in eclogites provides the retrograde path of the host rocks. The latest metamorphism of this complex is of the epidote amphibolite facies.

The garnet-clinopyroxene geothermometer of eclogites gives a lower temperature compared with the exhumation path of the host rocks, although the eclogites are of the low temperature type. Eclogite with type I garnet (93070110, garnet mantle) underwent UHPM at $\sim 510^{\circ} \mathrm{C}$ (Table 4). HPM eclogite with type I garnet (93062901B, garnet mantle) crystallized at $\sim 405{ }^{\circ} \mathrm{C}$. The metamorphic temperature of HPM eclogites with type II garnet (34$53 \mathrm{~A}$, garnet mantle) and type III garnet (82-2641) ranges from $\sim 290{ }^{\circ} \mathrm{C}$ to $510^{\circ} \mathrm{C}$. The $P-T$ conditions of these three eclogites are in the low temperature range, outside of the stability field of chloritoid + talc (Wei and Powell, 2003). One eclogite (94071610, garnet mantle) only gives $\sim 580{ }^{\circ} \mathrm{C}$ (or $660{ }^{\circ} \mathrm{C}$ ), which is in the stability field of chloritoid + talc.

Moreover, the metamorphic age of the exotic blocks ranges from 769 to $881 \mathrm{Ma}$; it is older than that of the host rock, viz., 480-509 Ma. Therefore, only mélange tectonics can explain this mixture of host rocks, eclogites, and exotic blocks. These geological relationships suggest that the ascending materials must be an alternation of pelitic and quartz schists. The alternation of pelitic and quartz schists has a lower density as compared to eclogites and peridotites. Accordingly, these strata always have significant buoyancy in the subduction wedge.

The low temperature type of coesite eclogite facies is formed under a very low geothermal gradient $\left(\sim 5{ }^{\circ} \mathrm{C} /\right.$ $\mathrm{km}$ ) at the subduction zone. There is a high possibility of the subducted substances being heated up because of the decrease in the descending speed and the cessation of subduction. The $P-T$ condition of the coesite schists reaches the transition boundary from coesite to quartz, and coesite immediately transforms into quartz. As the volume of the ascending strata increases instantaneously, the buoyancy force increases, as mentioned by Dobretsov (1991) and Ernst and Liou (1995).
The mass transition from coesite to quartz may be one of the triggers for the high-speed exhumation of wedge material as a solid intrusion. During the ascension of UHPM rocks, the host rocks have a high probability of capturing eclogites that were present in the passage route of the ascending host rocks. This produces eclogite lenses of the low temperature type HPM or of the Makbal UHPM. The high-speed exhumation results in the formation of eclogite lenses of the low temperature type of UHPM. At higher exhumation speeds, the ascending host rocks intrude into the crust and are able to capture pre-existing HPM rocks as exotic blocks. Deformation structures such as slip plane, imbrication, and drag fold of eclogite lenses were formed during coupling. The corresponding older HPM rocks are outcropped in the Aktyuz area, northern Tien-Shan (Tagiri et al., 1995; Orozbaev et al., 2007).

We propose the following ascension scenario. The Makbal UHP host rocks captured many HP eclogite lenses belonging to the Makbal Complex at about $0.8-1.3 \mathrm{GPa}$ under the low-temperature condition of the quartz eclogite facies; they also captured many exotic blocks at less than $0.8 \mathrm{GPa}$ under the low-temperature condition of epidote amphibolite facies. The present imbricated structure of the eclogite body was formed during ascension through the crust.

\section{ACKNOWLEDGMENTS}

Prof. Watanabe is posthumously acknowledged as an important cooperative researcher of this study. We are grateful to Prof. W.G. Ernst and late Prof. S. Banno for their encouragement and advice. We also thank E.A. Ivleva and D. Ukudeeva for their assistance in the field survey, and Dr. A. Fujinawa, Dr. A. Goto, and K. Murayama for their cooperation. We acknowledge the collaboration by Prof. H. Tanaka, P. Puelles, Y. Mochi, T. Yano, A. Takenaka, and K. Takamura. We are indebted to K. Kawakami and Y. Sugawara of the Mitsubishi Materials Corporation for the $\mathrm{K}$-Ar age dating. Many comments by three referees, especially Prof. T. Hirajima and Prof. A. Korsakov, were extremely useful in helping us improve the manuscript. This study was partly supported by the Japan Society for the Promotion of Science (1993-94), by a Grant-in-Aid for Scientific Research (B) (Takasu, No.17340149), and by the National Academy of Sciences, Kyrgyz Republic.

\section{REFERENCES}

Bakirov, A., Tagiri, M. and Sakiev, K. (1998) Rocks of ultrahighpressure metamorphic facies in the Tien Shan. Russian Geology and Geophysics, 39, 1709-1721. 
Bakirov, A.B., Biske, Ju.S., Burtman, V.S., Chernyshuk, V.P., Dzhumagulov, A.D., Kashirin, F.T., Khristov, E.B., Klishevich, V.L, Kurenkov, S.A., Osmonbetov, K.O., Rogozhin, E.A. and Vanina, L.V. (1984) Guide book for excursion 032: Tectonics of the Tien Shan Varicides. 27th session, IGC, Moscow $1984,74$.

Banno, S. (1959) Aegirine-augite from crystalline schists in Sikoku. Journal of the Geological Society of Japan, 65, 652-657.

Bence, A.E. and Albee, A.L. (1968) Empirical correction factors for the electron microanalysis of silicates and oxides. Journal of Geology. 76, 382-403.

Brown, E.H. (1977) The crossite content of Ca-amphibole as a guide to pressure of metamorphism. Journal of Petrology, 18, 53-72.

Carswell, D.A. (1990) Eclogites and the eclogite facies: definitions and classification. In Eclogite Facies Rocks( Carswell, D.A. Ed.). Blackie, London, 1-13.

Carswell, D.A. and Harley, S.L. (1990) Mineral barometry and thermometry. In Eclogite Facies Rocks (Carswell, D.A. Ed.). Blackie, London, 83-110.

Chemenda, A., Matte, P. and Sokolov, V. (1997) A model of Palaeozoic obduction and exhumation of high-pressure/lowtemperature rocks in the southern Urals. Tectonophysics, 276, 217-227.

Dalrymple, G.B. and Lanphere, M.A. (1969) Potassium-Argon Dating. pp. 258, Freemann, San Francisco.

Dobretsov, N.L. (1991) Blueschists and eclogites: a possible plate tectonic mechanism for their emplacement from the upper mantle. Tectonophysics, 186, 253-268.

Dobretsov, N.L. and Shatsky, V.S. (2004) Exhumation of highpressure rocks of Kokchetav Massif: facts and models. Lithos, $78,307-318$

Duchêne, S., Blichert-Toft, J., Luais, B., Telouk, P., Lardeaux, J.M. and Albarede, F. (1997) The Lu-Hf dating of garnets and the ages of the Alpine high-pressure metamorphism. Nature, 387, 586-589.

Editorial Committee of Geological Society of Japan (2000) Propose the mineral abbreviation in Journal of the Geological Society of Japan. Journal of the Geological Society of Japan, 106, No.10 and 11, Cover pages.

Ellis, D.J. and Green, D.H. (1979) An experimental study of the effect of $\mathrm{Ca}$ upon garnet-clinopyroxene $\mathrm{Fe}-\mathrm{Mg}$ exchange equilibria. Contributions to Mineralogy and Petrology, 71, 13-22.

England, P.C. and Holland, T.J.B. (1979) Archimedes and the Tauern eclogites: The role of buoyancy in the preservation of exotic eclogite blocks. Earth and Planetary Science Letters, 44, 287-

Ernst, W.G. and Liou, J.G. (1995) Contrasting plate-tectonic styles of the Qinling-Dabie-Sulu and Franciscan metamorphic belts. Geology, 23, 353-356.

Gebauer, D., Schertle, H.-P., Brix, M. and Schreyer, W. (1997) 35 Ma old ultrahigh-pressure metamorphism and evidence for very rapid exhumation in the Dora Maira Massif, Western Alps. Lithos, 41, 5-24.

Hacker, B.R. and Peacock, S.M. (1995) Creation, preservation, and exhumation of UHPM rocks. In Ultrahigh Pressure Metamorphism (Coleman, R.G., Wang, X. Eds.). Cambridge University Press, Cambridge, New York, 159-181.

Hacker, B.R., and Wang, Q.C. (1995) Ar/Ar geochronology of ultrahigh-pressure metamorphism in central China, Tectonics,
14, 994-1006.

Hermann, J., Rubatto, D. and Korsakov, A. and Shatsky V.S. (2001) Multiple zircon growth during fast exhumation of diamondiferous, deeply subducted continental crust (Kokchetav Massif, Kazakhstan). Contributions to Mineralogy and Petrology, 141, 66-82.

Hirajima, T., Banno, S., Hiroi, Y. and Ohta, Y. (1988) Phase petrology of eclogites and related rocks from the Motalafjella high-pressure metamorphic complex in Spitsbergen (Arctic Ocean) and its significance. Lithos, 22, 75-97.

Hirajima, T. and Nakamura, D. (2003) The Dabie Shan-Sulu orogen. EMU Notes in Mineralogy, 5, 105-144.

Holland, T.J.B. (1980) The reaction albite $=$ jadeite + quartz determined experimentally in the range $600-1200{ }^{\circ} \mathrm{C}$. American Mineralogist, 65, 125-134.

Holland, T.J.B. and Richardson, S.W. (1979) Amphibole zonation in metabasites as a guide to the evolution of metamorphic condition. Contributions to Mineralogy and Petrology, 70, 143-148.

Kaneko, Y., Maruyama, S., Terabayashi, M., Yamamoto, H., Ishikawa, M., Anma, R., Parkinson, C.D., Ota, T., Nakajima, Y., Katayama, I., Yamamoto, J. and Yamauchi, K. (2000) Geology of the Kokchetav UHP-HP metamorphic belt, Northern Kazakhstan. The Island Arc, 9, 264-283.

Katayama, I., Maruyama, S., Parkinson, C.D., Terada, K. and Sano, S. (2001) Ion micro-probe U-Pb zircon geochronology of peak and retrograde stages of ultrahigh-pressure metamorphic rocks from the Kokchetav massif, northern Kazakhstan. Earth and Planetary Science Letters, 188, 185-198.

Kennedy, C.S. and Kennedy, G.C. (1976) The equilibrium boundary between graphite and diamond. Journal of Geophysical Research, 81, 2467-70.

Krogh, E.J. (1988) The garnet-clinopyroxene Fe-Mg geothermometer- a reinterpretation of existing experimental data. Contributions to Mineraloty and Petrology, 99, 44-48.

Liu, L., Mernagh, T. P. and Hibberson, W. O. (1997) Raman spectra of high-pressure polymorphs of $\mathrm{SiO}_{2}$ at various temperatures. Physics and Chemistry of Minerals, 24, 396-402.

Massonne, H.J. (1995) Experimental and petrogenetic study of UHPM. InUltrahigh Pressure Metamorphism (Coleman, R.G. and Wang, X. Eds.). Cambridge University Press, Cambridge, New York, 33-95.

Massonne, H.-J. and Schreyer, W. (1987) Phengite geobarometry based on the limiting assemblage with $\mathrm{K}$-feldspar, phlogopite and quartz. Contributions to Mineralogy and Petrology, 96, 212-224.

Massonne, H.-J. and Schreyer, W. (1989) Stability field of the high-pressure assemblage talc + phengite and two new phengite barometers. European Journal of Mineralogy, 1, 391410.

Mirwald, P.W. and Massonne, H.J. (1980) The low-high quartz and quartz-coesite transition to $40 \mathrm{kbar}$ between $600{ }^{\circ} \mathrm{C}$ and $1600{ }^{\circ} \mathrm{C}$ and some reconnaissance data on the effect of $\mathrm{NaAlO}_{2}$ component on the low quartz-coesite transition. Journal of Geophysical Research, 85, B12, 6983-6990.

Orozbaev, R.T., Takasu, A., Tagiri, M., Bakirov, A.B. and Sakiev, K.S. (2007) Polymetamophism of Aktyuz eclogites (northern Kyrgyz Tien-Shan) deduced from inclusion in garnets. Journal of Mineralogical and Petrological Sciences, 102, 150156.

Powell, L R. (1985) Regression diagnostics and robust regression in geothermometer/ geobarometer calibration: the garnet 
clinopyroxene geothermometer revisited. Journal of Metamorphic Geology, 3, 231-243.

Platt, J.P. and Wallis, S.R. (1991) Did the high-pressure metamorphic rocks exhume by what kind of mechanism? Kagaku, 61, 535-543 (In Japanese).

Puelles, P. and Takasu, A. (1996) Chemical compositions of the constituent minerals of the Kyrghyzstan high-P and ultrahigh-P metamorphic rocks. Geoscience Report of Shimane University, 15, 115-135.

Rubatto, D. and Hermann, J. (2001) Exhumation as fast as subduction? Geology, 29, 3-6.

Rumble, D., Liou, J.G. and Jahn, B.M. (2005) Continental crust subduction and ultrahigh pressure metamorphism. In the Crust (R.L. Rudnick Ed.). Elsevier, 293-319.

Sobolev, N.V., Dobretsov, N.L., Bakirov, A.B. and Shatsky, V.S. (1986) Eclogites from various types of metamorphic complexes in the USSR and the problems of their origin. Geological Society of America, Memoir 164, 349-63.

Steiger, R.H. and Jäger, E. (1977) Subcommission on geo-chronology: convention on the use of decay constants in geo- $^{-}$and cosmochronology. Earth and Planetary Science Letters, 36, 359-362.

Tagiri, M. and Bakirov, A. (1990) Quartz pseudomorph after coesite in garnet from a garnet-chloritoid-talc schist, Northern Tien-Shan, Kirghiz SSR. Proceedings of the Japan Academy, 66, 135-39.

Tagiri, M., Yano, T., Bakirov, A., Nakajima, T. and Uchiumi, S. (1995) Mineral parageneses and metamorphic P-T paths of ultrahigh-pressure eclogites from Kyrghyzstan Tien-Shan. The Island Arc, 4, 280-292.

Togonbaeva, A.A., Takasu, A., Bakirov, A.A., Sakurai, T., Tagiri, M., Bakirov, A.B. and Sakiev, K.S. (2009) U-Th-Pb CHIME ages of monazites from garnet-chloritoid-talc schists in the Makbal Complex, Northern Kyrgyz Tien-Shan: First report of the age of the UHP metamorphism. Journal of Mineralogical and Petrological Sciences, 104, 77-81.

Wei, C. and Powell, R. (2003) Phase relations in high-pressure metapelites in the system KFMASH $\left(\mathrm{K}_{2} \mathrm{O}-\mathrm{FeO}-\mathrm{MgO}-\right.$ $\mathrm{Al}_{2} \mathrm{O}_{3}-\mathrm{SiO}_{2}-\mathrm{H}_{2} \mathrm{O}$ ) with application to natural rocks. Contributions to Mineralogy and Petrology, 145, 301-315.

Wei, C. and Powell, R. (2004) Calculated phase relations in highpressure metapelites in the system NKFMASH $\left(\mathrm{Na}_{2} \mathrm{O}-\mathrm{K}^{2} \mathrm{O}-\right.$ FeO-MgO- $\mathrm{Al}_{2} \mathrm{O}_{3}-\mathrm{SiO}_{2}-\mathrm{H}_{2} \mathrm{O}$ ). Journal of Petrology, 45, 183202.

Wei, C. and Song S.G. (2008) Chloritoid-glaucophane schist in the north Qilian orogen, NW China: phase equilibria and P-T path from garnet zonation. Journal of Metamorphic Geology, 26, 301-316.

Yang, J.S., Xu, Z., Song, S., Zhang, J., Shi, R., Li, H. and Brunel, M. (2001) Discovery of coesite in the North Qaidan Early Paleozoic ultrahigh pressure (UHP) metamorphic belt, NW China. Comptes Rendus de l'Academie des Sciences, Paris, Sciences de la Terre et des Planets, 333, 719-724.

Zhang, R.Y., Liou, J.G., Ernst, W.G., Coleman, R.G., Sobolev, N.V. and Shatsky, V.S. (1997) Metamorphic evolution of diamond-bearing and associated rocks from the Kokchetav Massif, northern Kazakhstan. Journal of Metamorphic Geology, 15, 479-496.

Manuscript received October 25, 2007

Manuscript accepted February 14, 2010

Published online May 19, 2010

Manuscript handled by Takao Hirajima 